\title{
ÁLGEBRAS DE $p$-GRUPOS COM BASES MULTIPLICATIVAS FILTRADAS
}

\author{
José Cloves Verde Saraiva
}

\author{
TESE APRESENTADA \\ $\mathrm{AO}$ \\ INSTITUTO DE MATEMÁTICA E ESTATÍSTICA \\ DA \\ UNIVERSIDADE DE SÃO PAULO \\ PARA \\ OBTENÇÃO DO GRAU \\ $\mathrm{DE}$ \\ DOUTOR EM MATEMÁTICA
}

Área de Concentração: Álgebra

Orientador: Prof. Dr. Francisco César Polcino Milies

- São Paulo, março de 1999 - 


\section{ÁLGEBRAS DE $p$-GRUPOS COM \\ BASES MULTIPLICATIVAS FILTRADAS}

José Cloves Verde Saraiva

Este exemplar corresponde à redação final da tese apresentada por José Cloves Verde Saraiva, devidamente corrigida e aprovada pela Comissão Julgadora

São Paulo, 22 de março de 1999

\section{Comissão Julgadora}

- Prof. Dr. Francisco César Polcino Milies - IME-USP

- Prof. Dr. Jairo Zacarias Gonçalves - IME-USP

- Prof. Dr. Rudolf Richard Maier - UnB

- Prof. Dr. Norai Rlomeu Rocco - UnB

- Prof. Dr. Guilherme Augusto de La Rocque Leal - UFRJ 


\section{RESUMO}

Seja $K$ um corpo de característica $p>0$ e $G$ um $p$-grupo finito metacíclico. Apresentamos neste trabalho uma classificação das álgebras de grupo $K G$ que possuem uma base multiplicativa filtrada sobre o corpo $K$. Apresentamos também uma aplicação da técnica empregada na demonstração do Teorema de Classificação para determinar bases multiplicativas filtradas de outras álgebras de grupo $K G$, onde $G$ é um p-grupo não-metacíclico.

\section{ABSTRACT}

Let $K$ be a field of the characteristic $p>0$ and let $G$ be a finite metacyclic $p$-group. We present a classification of the group algebras $K G$ which have a multiplicative filtered base over $K$. We also present an application of the technique used in the proof of this classification to determine such bases for others $K G$ where $G$ are not metacyclic. 
..., but I'm gonna wave my freak flag high, high.

(If six was nine - James M. Hendrix) 
Tudo aqui escrito é dedicado à memória do meu avô, Eduardo Otelo da Silva Coqueiro, e daqueles que saudosamente partiram mas ainda vivem na minha lembrança.

I'ma dedicatória especial aos meus pais, Maria José e José da Paz Saraiva, e à minha querida esposa, Maria Vitória e aos filhos Andrei e Raysa, e à minha avó Felícia nos seus 90 anos. 


\section{AGRADECIMENTOS}

Diante do trabalho a fé, a esperança alcançada e os corações de muitos, não os esquecerei. Só por DEUS! conceder tudo o quanto até agora escrevo. E, neste momento, tento olhar pra todo o caminho que me conduziu a esta formação e ver a "gente boa" acreditando na minha força de vontade e me dar a chance de concluir meu objetivo. Resta-me agradecer.

Inicialmente, agradeço aos colegas Inaldo Tavares, José de Ribamar Cabral Lopes, Francisco Pinheiro Dominice, Cristian Novoa Bustos, Ezio Costa, João Batista Nascimento, Andreas Brunner, Luciano Huanca e Rosângela Kulcsar pelo que no dia a dia o estudo proporcionou agradáveis encontros e fraterna amizade.

Aqui registro os meus agradecimentos aos queridos mestres, Profas. Helena Santos e Dilsa Furtado, Profs. Raymundo Moacir, Eleazar Carvalho, Olavo Correia Lima, Rudolf Richard Maier. Agradeço também ao Prof. Chaim Hönig pelas conversas e sutilezas gerais e não esqueço de agradecer o encorajamento dos professores Plínio Simões, Rui Tavares, Jairo Gonçalves, Luíz Gonzaga Xavier de Barros, Orlando Stanley, Nair Fernandes, Hector Merklen e especialmente aos professores visitantes Victor Bovdi e seu pai, A.A. Bovdi, por sugerirem o tema. O Teorema 3.1.1 foi obtido em colaboração com V. Bovdi.

Ao professor Dr. Francisco César Polcino Milies cabe o meu maior agradecimento, por me aceitar como aluno e fundamentalmente por sua sábia orientação, sugestões, correções, aceitar o estudo deste problema e por acreditar no meu trabalho.

Finalmente, agradecer aos que com grande empenho fazem a infra-estrutura do IMEUSP, os amigos da limpeza, segurança, o bom café e ao bom pessoal da Biblioteca Marina, Sílvia, Inácia, Eunice, Alessandra, Nádia, Luísa, Célia e Teresa -, Marlene da Xerox e os amigos do restaurante Crisma. Também aos colegas da UFMA, em especial Maria Luiza, Vera Lobato, Durval Rocha, Maria Rita, Jocelino Melo, José Romão e Luis Carlos Filgueiras de Carvalho e aos amigos Amorim Pinto, Wilson Passos, Ribinha e Arimatéia, pela valiosa colaboração, e ao Walter, pelo esmero na digitação.

À glória de São Luís, São Paulo, dos milagres de São José de Ribamar e ao sol da Praia do Araçagi. 


\section{Conteúdo}

1 Introdução 1

1.1 Bases multiplicativas filtradas para $p$-grupos abelianos . . . . . . . . 3

2 Resultados básicos e notações $\quad 6$

2.1 Grupos $($-grupos metacíclicos $) \ldots \ldots \ldots$. . . . . . . . . 7

2.2 Álgebras de dimensão finita . . . . . . . . . . . . . . . . . . 9

$2.3 K$-bases multiplicativas filtradas - propriedades fundamentais . . . . . . . . 11

2.4 Álgebras de grupo . . . . . . . . . . . . . . . . . . . . 13

2.4 .1 Álgebras de $p$-grupos . . . . . . . . . . . . . . . . . . . . . 14

3 Bases multiplicativas filtradas para p-grupos metacíclicos finitos 28

3.1 Introdução . . . . . . . . . . . . . . . . . . . . . . . 28

3.2 O teorema de classificação . . . . . . . . . . . . . . . . . . . 34

4 Exemplo de p-grupo não metacíclicos com base multiplicativa filtrada 57

4.1 Exemplos especiais de álgebra de grupo com base multiplicativa filtrada . . 58

$\begin{array}{ll}\text { Referências } & 76\end{array}$ 


\section{Capítulo 1}

\section{Introdução}

O objetivo deste trabalho é resolver parcialmente a questão proposta por R. Bautista, P. Gabriel, A. Roiter e L. Salmeron na referência [1], que é a seguinte: Quando pode existir uma $K$-base multiplicativa filtrada numa álgebra de grupo $K G$ ? Sobre esta questão apenas L. Paris [18] dá exemplos de álgebras de grupo $K^{\prime} G$, que não possuem $K^{\prime}$-base multiplicativa filtrada e também prova que se $K$ é um corpo de característica 2 e $G$ o 2-grupo diedral finito ou $G$ o grupo quatérnio com oito elementos e $K$ contendo uma raiz cúbica primitiva da unidade, então $K G$ tem uma $K$-base multiplicativa filtrada.

A resposta aqui apresentada trata da classificação das álgebras de grupo $K G$ de um p-grupo finito metacíclico $G$ sobre um corpo $K$ de característica $p>0$ tais que $K G$ possui uma $K$-base multiplicativa filtrada. Nossa resposta está escrita no Capítulo 3, no Teorema 3.1.1, onde somente as álgebras de grupo dadas como exemplo por L. Paris [18] têm uma tal $K$-base.

No capítulo 4 trabalhamos numa situação mais geral do que tratamos no Teorema 3.1.1, 
isto é, tratamos com p-grupos não metacíclicos, onde apresentamos um novo $p$-grupo com base multiplicativa filtrada, e também apresentamos outros $p$-grupos três geradores sem base multiplicativa filtrada.

Para melhor entendimento do assunto, fazemos a seguinte definição:

Definição 1.0.1 Seja A uma álgebra de dimensão finita sobre um corpo $K$ e considere o conjunto $B$ uma $K$-base de $A$. Dizemos que $B$ é uma $K$-base multiplicativa filtrada se valem as seguintes propriedades:

1) $B \cup\{0\}$ é um semigrupo para multiplicação de $A$;

2) $B \cap \operatorname{rad}(A)$ é uma $K$-base do $\operatorname{rad}(A)$, onde $\operatorname{rad}(A)$ denota o radical de Jacobson de A;

3) Se $e, e^{\prime} \in B \backslash \operatorname{rad}(A), e \neq e^{\prime}$, então $e^{2}=e \quad e \quad e . e^{\prime}=0$.

Estas $K$-bases especiais foram introduzidas por H. Kupisch, em 1965, tratando da Teoria das Representações de Álgebras [14]. 


\subsection{Bases multiplicativas filtradas para $p$-grupos abe- lianos}

Os resultados citados nesta seção são já conhecidos, devidos a L. Paris [18]. Devido ao teorema de estrutura dos p-grupos abelianos finitos, os seguintes resultados são fundamentais à construção de bases multiplicativas filtradas para qualquer $p$-grupo abeliano.

Resultado 1.1.1 Seja $G$ um p-grupo cíclico finito, $G=\left\langle a: a^{p^{n}}=1\right\rangle, n \geq 1$ e $K$ um corpo de características $p>0$. Então o conjunto

$$
B=\left\{(a-1)^{k} \mid 0 \leq k<p^{n}\right\}
$$

é uma $K$-base multiplicativa filtrada da álgebra $K G$.

Resultado 1.1.2 Sejam $K G_{1}$ e $K G_{2}$ álgebras de grupo com $K$-bases multiplicativas filtradas $B_{1}, B_{2}$, respectivamente, então $B_{1} \times B_{2}$ é uma $K$-base multiplicativa filtrada de $K\left[G_{1} \times G_{2}\right]$.

Imediatamente segue que:

Resultado 1.1.3 Seja $G=\left\langle a_{1}\right\rangle \times \cdots \times\left\langle a_{s}\right\rangle$ um p-grupo abeliano finito escrito como produto direto de subgrupos cíclicos $\left\langle a_{i}\right\rangle$ de ordem $q_{i}, 1 \leq i \leq s$, então o conjunto

$$
B=\left\{\left(a_{1}-1\right)^{n_{1}}\left(a_{2}-1\right)^{n_{2}} \cdots\left(a_{s}-1\right)^{n_{s}} \mid 0 \leq n_{i}<q_{i}\right\}
$$

é uma $K$-base multiplicativa filtrada da álgebra $K G$ sobre o corpo $K$ de característica $p>0$. 
Neste trabalho trataremos exclusivamente com álgebras de grupo $K G$, onde $K$ um corpo de característica $p>0$ e o grupo $G$ é um p-grupo finito. Na página 16, como conseqüência do Resultado 2.4.2(1), provaremos que toda $K$-base de $K G$ verifica trivialmente a condição 3$)$.

Na referência [1], R. Bautista, P. Gabriel, A. Roiter e L. Salmeron provaram que se A é uma álgebra tipo representação finita (isto é, no qual existe somente um número finito de classes de isomorfismo de $A$-módulo indecomponíveis) sobre um corpo $K$ algebricamente fechado, então $A$ tem uma $K$-base multiplicativa filtrada.

O seguinte teorema, devido a G. Higman, nos diz como são as álgebras de grupo em geral que são do tipo representação finita. Vide [5, Teorema 5.13, p.57].

Teorema 1.1.4 (G. Higman) Uma álgebra de grupo $K G$ do grupo $G$ sobre um corpo $K$ de característica $p>0$ é do tipo representação finita se, e somente se, os p-subgrupos de Sylow de G são cíclicos.

Importante é que a existência das bases multiplicativas filtradas não caracteriza uma álgebra de grupo tipo representação finito.

A álgebra de grupo $K\left(C_{p} \times C_{p}\right), p \geq 3$, onde o grupo é o produto direto de grupos cíclicos de ordem $p$ e $K$ um corpo de característica $p>0$ é um exemplo de álgebra de grupo não do tipo representação finita (pelo Teorema de Higman), mas pelo Resultado 1.1.3 iem uma $K$-base multiplicativa filtrada. 
Finalizamos esta introdução informando que P. Lendrock e G.O. Michler [16] provaram que a álgebra de grupo do menor grupo de Janko, $J_{1}$, sobre um corpo de característica 2 não possui uma $K$-base multiplicativa filtrada. 


\section{Capítulo 2}

\section{Resultados básicos e notações}

Neste capítulo descrevemos os resultados, definições, e introduzimos a notação a ser usada no contexto deste trabalho. Para melhor adequar as informações, este ficará dividido em subseções, onde começamos com grupos, tratando especialmente dos p-grupos e mais particularmente dos p-grupos metacíclicos. Dando continuidade, tratamos das álgebras de dimensão finita, suas bases, subálgebras importantes, os ideais e especialmente o radical de Jacobson e, a seguir, numa nova subseção tratamos das álgebras de grupo, principalmente das álgebras de grupo $K G$ de um $p$-grupo $G$ sobre um corpo $K$ de característica $p>$ 0 . Sobre estas álgebras de grupo de um $p$-grupo tratamos da determinação de $K$-bases para certos quocientes de portência do radical, como caso especial do que já foi tratado por Jennings [9]. Para maior entendimento, também, ou fazemos uma demonstração do resultado apresentado ou indicamos a referência onde este se encontra. 


\subsection{Grupos (p-grupos metacíclicos)}

Neste trabalho todos os grupos são finitos e os resultados principais aqui tratados são com respeito à classe dos p-grupos onde $p$ é um número primo.

Definição 2.1.1 Dizemos que um grupo $G$ é metacíclico se este tem um subgrupo normal H tal que $H$ e $G / H$ são cíclicos.

Os principais exemplos são os diedrais, semi-diedrais, quatérnios generalizados etc.

Nesta classe de grupos, no caso de $G$ ser um grupo finito, podemos estabelecer que

$$
H=\langle a\rangle \approx \mathbb{Z}_{m} \quad \text { e } \quad \frac{G}{H}=\langle H b\rangle \approx \mathbb{Z}_{n},
$$

com $a, b \in G$ e $m, n \in \mathrm{N}$. Desde que o índice de $H$ é $n$, temos que $b^{n} \in H$ e $b^{-1} a b \in H$, o que nos permite escrever

$$
b^{-1} a b=a^{r} \quad \text { e } \quad b^{n}=a^{s},
$$

onde $r, s$ são números inteiros, $1 \leq r, s \leq m$. Daí, é fácil ver que $b^{-1} a^{t} b=a^{t r}, \operatorname{logo}$ $b^{-k} a^{t} b^{k}=a^{t r^{k}}$. Então, por ser $b^{n}=a^{s}$, temos

$$
a=a^{-s} a a^{s}=b^{-n} a b^{n}=a^{r^{n}},
$$

portanto, $r^{n} \equiv 1(\bmod m)$, pois $o(a)=|H|=m$. Analogamente vale $a^{s}=b^{n}=b^{-1} b^{n} b^{-1}=$ $b^{-1} a^{s} b=a^{r s}$, portanto, $r s \equiv s(\bmod m)$.

Estas observações permitem escrever uma apresentação para todo grupo metacíclico finito. Particularmente, para um p-grupo metacíclico, os seguintes resultados podem ser encontrados em [8, p.336, Satz 11.2]: 
Resultado 2.1.2 Todo p-grupo metacíclico $G$ admite a seguinte apresentação:

$$
G=\left\langle a, b \mid a^{p^{n}}=1, b^{p^{m}}=a^{p^{t}},{ }^{b} a=a^{r}\right\rangle,
$$

onde $t \geq 0, r^{p^{m}} \equiv 1\left(\bmod p^{n}\right)$ e $p^{t}(r-1) \equiv 0\left(\bmod p^{n}\right)$. Portanto, todo elemento $g \in G$ pode ser escrito com $g=a^{i} b^{j}$, onde $0 \leq i \leq p^{n}$ e $0 \leq j<p^{m}$ e claramente $|G|=p^{n+m}$.

Observa-se que na apresentação de $G$, a notação da conjugação ${ }^{b} a=b a b^{-1}$ e usamos para o comutador $(b, a)={ }^{b} a a^{-1}=b a b^{-1} a^{-1}$ (estas notações são introduzidas na referência $[8])$.

Também é conhecido o seguinte resultado:

Resultado 2.1.3 Sendo $G$ um p-grupo não abeliano, então o fator $G / G^{\prime}$ não pode ser cíclico.

Uma demonstração sai da caracterização de Wielandt que o subgrupo comutador de $G$, $G^{\prime}$, é um subgrupo do subgrupo de Frattini, $\Phi(G)$, de $G$, vale em geral para grupos nilpotentes aqui em particular para $p$-grupo e do Teorema da Base de Brunside [8, Hilfssatz 7.1c, p.300].

Concluímos esta seção introduzindo alguma notação. Sejam $H, K$ subgrupos de um grupo qualquer $G$. Definimos

$$
\begin{aligned}
& {\left[H, K^{\prime}\right]=\left\langle(h, k), h \in H, k \in K^{\prime}\right\rangle } \\
\text { e } \quad & G^{p}=\left\langle x^{p} \mid x \in G\right\rangle .
\end{aligned}
$$




\section{2 Álgebras de dimensão finita}

Seja $A$ uma álgebra de dimensão finita sobre um corpo $K$. Então seus elementos são dados por combinações lineares, $a=\sum_{i=1}^{n} a_{i} \varepsilon_{i}$ de $n$ elementos básicos $\varepsilon_{1}, \varepsilon_{2}, \ldots, \varepsilon_{n} \quad \mathrm{e}$ coeficientes $a_{i} \in K$. Daí, naturalmente, definimos a adição, a multiplicação por escalares e o produto dado pela fórmula

$$
\left(\sum_{i=1}^{n} a_{i} \varepsilon_{i}\right)\left(\sum_{i=1}^{n} b_{i} \varepsilon_{i}\right)=\sum_{i, j=1}^{n}\left(a_{i} b_{j}\right) \varepsilon_{i} \varepsilon_{j},
$$

onde é conhecido o produto dos elementos básicos $\varepsilon_{i} \varepsilon_{j}$ e assim a multiplicação e a álgebra $A$ ficam determinadas com respeito à base $B=\left\{\varepsilon_{1}, \ldots, \varepsilon_{n}\right\}$.

Neste trabalho, tratamos de conceitos bastante conhecidos no estudo das álgebras, e destacamos os seguintes com respeito à álgebra $A$ : subálgebra de $A$, ideais à esquerda (à direita), ideais bilaterais e as álgebras quocientes. Dentre estes conceitos, recordamos agora os mais importantes.

Definição 2.2.1 A subálgebra de Frattini de A, denotada por $\Phi(A)$, é definida como a interseçãa de todas as subálgebras maximais de A, se estas existem; caso contrário, define-se $\Phi(A)=A$.

Definição 2.2.2 Dizemos que um elemento $a \in A$ é nilpotente se existe um número inteiro positivo $k$ tal que $a^{k}=0$.

Definição 2.2.3 Sejam I e J ideais à esquerda (à direita, bilaterais), o subgrupo aditivo de A gerado pelos produtos ab onde $a \in I, b \in J$ e denotado por $I J$ é um ideal à esquerda 
(à direita, bilateral) de A. E, assim, indutivamente, definimos as potências de I por

$$
I^{n}=I^{n-1} I \quad \text { onde } \quad I^{1}=I .
$$

Observa-se que $I^{n}$ consiste de todas as somas finitas

$$
\sum_{i=1}^{r} a_{i_{1}} a_{i_{2}} \cdots a_{i_{n}}, \quad \text { com } a_{i_{j}} \in I \quad \text { e } \quad r \geq 1 .
$$

Definição 2.2.4 Dizemos que o ideal I de A é nilpotente se alguma potência de I é zero. $O$ menor inteiro positivo $k$ que satisfaz $I^{k}=0$ é chamado o índice de nilpotência do ideal I. Particularmente, se $I=A$ é nilpotente, dizemos que A é uma álgebra nilpotente.

Destacamos um resultado importante de G.L. Carns e C.Y. Chao (vide [2]):

Resultado 2.2.5 Seja A uma álgebra de dimensão finita nilpotente, então $\Phi(A)=A^{2}$.

São conhecidos os seguintes resultados, que podem ser encontrados em [4] e [5]:

Resultado 2.2.6 Seja A uma álgebra de dimensão finita sobre um corpo $K$ de dimensão $n$ e seja I um ideal lateral de A. Se todo elemento de I é nilpotente, então I é nilpotente e o índice de nilpotência de I é no máximo $n+1$.

Resultado 2.2.7 Seja A uma álgebra de dimensão $n$ sobre um corpo $K$. Se existe uma $K$-base de A formada só por elementos nilpotentes, então a própria A é uma álgebra nilpotente (vide [7]). 
Definição 2.2.8 Seja A uma.álgebra de dimensão finita sobre um corpo. O radical de Jacobson de A, denotado por $\operatorname{rad}(A)$, é definido como a intersecção de todos os ideais à esquerda maximais de $A$, se estes existirem; caso contrário, define-se $\operatorname{rad}(A)=A$.

Neste trabalho o conceito principal de $K$-base multiplicativa filtrada de uma $K$-álgebra depende especialmente de $\operatorname{rad}(A)$, isto é, do radical de Jacobson da álgebra $A$. Por isso, finalizamos este subitem com o

Resultado 2.2.9 Seja A uma álgebra de dimensão finita sobre um corpo $K$, então valem os seguintes itens:

(a) $\operatorname{rad}(A)$ é nilpotente;

(b) $\operatorname{rad}(A)$ contém todos os ideais à esquerda nilpotentes de $A$;

(Vide referência [5].)

\section{3 $K$-bases multiplicativas filtradas - propriedades fundamentais}

Vamos provar nesta seção uma proposição fundamental no estudo e desenvolvimento do teorema principal deste trabalho. 
Proposição 2.3.1 Seja B uma base multiplicativa filtrada de uma álgebra de dimensão finita A sobre um corpo $K$. Então valem as seguintes propriedades:

(i) $B \cap \operatorname{rad}(A)^{n}$ é uma base do $\operatorname{rad}(A)^{n}, \forall n \geq 1$, sobre o corpo $K$;

(ii) Se $u, v \in B \backslash \operatorname{rad}(A)^{k}$ e $u \equiv v\left(\bmod \left(\operatorname{rad}(A)^{k}\right)\right)$, então $u=v$;

(iii) Se $B \backslash\{1\} \subseteq \operatorname{rad}(A)$, então $B \backslash \operatorname{rad}(A)^{2}$ são geradores de $A$ como álgebra sobre $K$.

\section{Demonstração:}

(i) Pela Definição $1.0 .1, B \cap \operatorname{rad}(A)$ é uma $K$-base do $\operatorname{rad}(A)$ e o subconjunto $B \cap$ $\operatorname{rad}(A)^{n}$ é linearmente independente sobre $K$. E, desde que o conjunto dos produtos $b_{1} b_{2} \cdots b_{n}, \operatorname{com} b_{i} \in B$ é um sistema de geradores para $\operatorname{rad}(A)^{n}$ e cada tal produto é sempre 0 ou pertence a $B \cap \operatorname{rad}(A)^{n}$, concluímos que $B \cap \operatorname{rad}(A)^{n}$ é uma $K$-base do $\operatorname{rad}(A)^{n}$.

(ii) Escrevendo $u-v=\sum_{w \in B \cap \operatorname{rad}(A)^{k}} \lambda_{w} w$, com $\lambda_{w} \in K$, temos pela independência linear dos elementos da base que todos os $\lambda_{w}$ são nulos e $u=v$.

(iii) Pelo Resultado 2.2.9, o radical de uma álgebra de dimensão finita $A$ é nilpotente e pela aplicaçào direta do Resultado 2.2 .5 particularmente aplicado ao $\operatorname{rad}(A) \mathrm{e}$ sabendo que a subálgebra de Frattini só contém não geradores. 


\section{4 Álgebras de grupo}

Seja $K$ um corpo e $G$ um grupo finito de ordem $|G|=n$. Se $g_{1}, g_{2}, \ldots, g_{n}$ são os elementos de $G$, considere $K G$ o conjunto de todas as somas formais

$$
\alpha_{1} g_{1}+\alpha_{2} g_{2}+\cdots+\alpha_{n} g_{n}=\sum_{i=1}^{n} \alpha_{i} g_{i}, \quad \operatorname{com} \alpha_{i} \in K
$$

onde duas tais expressòes, $\sum \alpha_{i} g_{i}$ e $\sum \beta_{i} g_{i}$ são consideradas iguais se e somente se $\alpha_{i}=\beta_{i}$, para todo $i=1,2, \ldots, n$. KG é um espaço vetorial definindo-se a adição e multiplicação por um escalar, usualmente dadas por.

1) $\sum_{i=1}^{n} \alpha_{i} g_{i}+\sum_{i=1}^{n} \beta_{i} g_{i}=\sum_{i=1}^{n}\left(\alpha_{i}+\beta_{i}\right) g_{i}$

2) $\lambda\left(\sum_{i=1}^{n} \alpha_{i} g_{i}\right)=\sum_{i=1}^{n}\left(\lambda \alpha_{i}\right) g_{i} \quad$ com $\lambda \in K$

e a multiplicação de $G$ induz uma multiplicação em $K G$, dada da seguinte forma:

$$
\left(\sum_{i=1}^{n} \alpha_{i} g_{i}\right)\left(\sum_{i=1}^{n} \beta_{i} g_{i}\right)=\sum_{k=1}^{n}\left(\sum_{g_{i} g_{j}=g_{k}} \alpha_{i} \beta_{j}\right) g_{k} .
$$

A identificação de $g_{i} \operatorname{com} 1 g_{i}, 1 \in K$, faz $G \subseteq K G$ e com esta identificação os elementos do grupo $G$ formam uma base de $K G$ sobre o corpo $K$. O conjunto $K G$ com as operações acima descritas é uma álgebra de dimensão finita $n$-dimensional sobre $K$ : é a chamada álgebra de grupo do grupo $G$ sobre o corpo $K$.

Sabe-se que $K G$ é uma álgebra com unidade e, pela identificação acima, a unidade é o elemento neutro do grupo, $1 \in G$ e claramente os elementos da $K$-base $G$ são invertíveis 
em $K G$. É também imediato ver que $K G$ é uma álgebra comutativa se e somente se $G$ é um grupo abeliano e, além disso, se $G \neq 1$ finito, $K G$ não pode ser uma álgebra de divisào.

Um resultado muito conhecido é o Teorema de Maschke: KG é semisimples se $e$ somente se a característica do corpo $K$ não divide a ordem de $G$.

Por outro lado, se car $K=p>0$ divide a ordem do grupo $G$, o radical de $K G$ é não nulo, pois basta ver que o ideal gerado por $\sigma=\sum_{i=1}^{n} g_{i} \in K G$ é de quadrado nulo, portanto, nilpotente e o Resultado 2.2 .9 garante $\operatorname{rad}(K G) \neq 0$.

Sabemos também que o homomorfismo de aumento $\varepsilon: K G \rightarrow K$ tem núcleo Ker $\varepsilon=$ $\left\{\sum_{i=1}^{n} \alpha_{i} g_{i} \in K G \mid \sum_{i=1}^{n} \alpha_{i}=0\right\}$, o ideal de aumento de $K G$, que aqui denotamos por $I_{K}(G)$.

Objetivando a prova do teorema principal deste trabalho, tratamos das álgebras de grupo modulares, particularmente com as álgebras $K G$, onde $G$ é um $p$-grupo finito e $K$ é um corpo de característica $p>0$.

Pela importância deste assunto, vamos introduzi-lo como uma subseção.

\subsection{1 Álgebras de $p$-grupos}

O básico neste estudo é o artigo famoso de Jennings (1941) [9], onde buscamos maior transparência no que se refere à determinação de $K$-bases para quocientes de potências do 
radical. Isto será feito no caso particular das álgebras de p-grupos metacíclicos modulares no teorema principal.

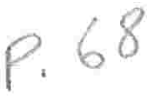

Resultado 2.4.1 (Jennings) O radical, $\operatorname{rad}(K G)$, da álgebra de grupo $K G$ de um $p$ grupo $G$ de ordem $p^{n}$ sobre um corpo $K$ de característica $p>0$ tem dimensão igual a $p^{n}-1$ e tem uma $K$-base formada por todos os elementos $g_{i}-1$, onde $1 \neq g_{i} \in G$.

O conjunto $\{g-1 \mid g \in G\}$ é uma base de $I_{K^{-}}(G)$ cujos elementos são todos nilpotentes. Pelo Resultado 2.2.7, segue que $I_{K^{-}}(G)$ é nilpotente e, pelo Resultado 2.2.9, temos que $I_{K^{*}}(G) \subset \operatorname{rad}\left(K^{*} G\right)$. Como $\operatorname{dim}\left(I_{K^{*}}(G)\right)=\operatorname{dim}\left(K^{\prime} G\right)-1$, segue que $I_{K^{\prime}}(G)=\operatorname{rad}\left(K^{K} G\right)$.

Pela nilpotência do $\operatorname{rad}(K G)=I_{K^{-}}(G)$, podemos escrever

$$
K G=I_{K^{\prime}}^{0}(a) \supset I_{K^{\prime}}^{1}(G) \supset I_{K^{(}}^{2}(G) \supset \cdots \supset I_{K^{(}}^{t}(G)=0
$$

onde $t=t(G)$ é o índice de nilpotência do radical de $K G$. Observamos que a seqüência decrescente de ideais (2.1) é comumente chamada de uma filtração do ideal $I_{K}(G)$ por ter a propriedade de que $I_{K^{(}}^{i}(G) I_{K^{-}}^{j}(G) \subseteq I_{K^{i}}^{i+j}(G)$ (vide referência [12], Teo.1.5(ii), p.308).

Agora destacamos os seguintes resultados sobre o índice de nilpotência $t=t(G)$, cujas demonstrações podem ser encontradas na referência [12, pp. 313-322].

Resultado 2.4.2 Seja G um p-grupo de ordem $p^{a}$, $a \geq 1$. Então valem:

1) D. Wallace (1968): $a(p-1)+1 \leq t(G) \leq p^{a}$; 
2) Motose-Ninomiya (1975):

(i) $t(G)=a(p-1)+1$ se e somente se $G$ é abeliano elementar;

(ii) $t(G)=p^{a}$ se e somente se $G$ é cíclico.

Agora, com este resultado, podemos provar que nas álgebras de grupo $K G$ onde $G$ é um $p$-grupo de ordem $p^{a}, a \geq 1$ e $K$ é um corpo de característica $p>0$, toda $K$ base $B$ de $K G$ verifica a condição (iii)) da Definição 1.0.1 pelo seguinte: se um elemento idempotente $e \in B \backslash \operatorname{rad}(K G)$, então $e=1+\xi \operatorname{com} \xi \in \operatorname{rad}\left(K^{\prime} G\right)$, pelo Resultado 2.4.1. Devido à idempotência de $e$, podemos escrever $e=e^{p^{a}}=(1+\xi)^{p^{a}}=1+\xi^{p^{a}}=1$, pois $\xi^{p^{a}}=0$ pelo item 1) do Resultado 2.4 .2 acima.

Concluímos que para um $p$-grupo $G$ sobre um corpo $K$ de característica $p>0$ a álgebra $K G$ é um anel local e seu único idempotente não nulo é 1 .

Resultado 2.4.3 (Koshitani (1977) e Motose (1978)) Se G é p-grupo metacíclico com apresentação

$$
G=\left\langle a, b \mid a^{p^{n}}=1, b^{p^{m}}=a^{p^{t}},{ }^{b} a=a^{r}\right\rangle,
$$

então

$$
t(G)= \begin{cases}p^{n}+p^{m}-1 & \text {, se } m \leq t \\ p^{n+m-t}+p^{t}-1 & \text {, se } m>t .\end{cases}
$$

Segue-se facilmente que os seguintes grupos de ordem $2^{n+1}$ possuem álgebras de grupo modular cujos radicais $I_{K}(G)$ têm índice de nilpotência igual a $2^{n}+1$. 
- Diedral: $G=\left\langle a, b \mid a^{2^{n}}=1, b^{2}=1,{ }^{b} a=a^{-1}\right\rangle$.

- Quatérnio Generalizado: $G=\left\langle a, b \mid a^{2^{n}}=1, b^{2}=a^{2^{n-1}},{ }^{b} a=a^{-1}\right\rangle$.

- Semidiedral: $G=\left\langle a, b \mid a^{2^{n}}=1, b^{2}=1,{ }^{b} a=a^{-1+2^{n-1}}\right\rangle$, que é isomorfo a

$$
G=\left\langle a, b \mid a^{2^{n}}=1, b^{2}=a^{2^{n-1}},{ }^{b} a=a^{-1+2^{n-1}}\right\rangle .
$$

Demonstração: Por serem metacíclicos $\operatorname{com} m=1$ e para o grupo diedral e semidiedral $t=n$, e $t=n-1$ para o grupo quatérnio generalizado, portanto temos que $1 \leq n-1<n$, pois $n \geq 2$. Aplicando diretamente o Resultado 2.4 .3 temos que

$$
t(G)=2^{n}+2^{1}-1=2^{n}+1 .
$$

Os $n$-ésimos subgrupos de dimensão de $G$ sobre o corpo $K$, se definem por

$$
\mathcal{D}_{n}(G)=G \cap\left(1+I_{K^{-}}^{n}(G)\right)(n \geq 1)
$$

e, por um resultado de Jennings, estes subgrupos característicos de $G$ podem ser computados recursivamente da seguinte forma:

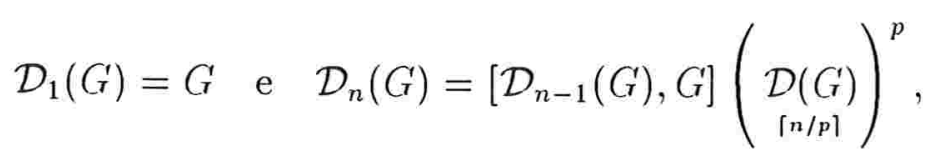

com $n \geq 2$, onde $\lceil n / p\rceil$ significa o menor inteiro maior do que ou igual à fração $n / p$ (vide referência [12, teo.1.6, p.309].

Resultado 2.4.4 Com a notação acima, vale que

$$
G^{\prime \prime}=\left[G, G^{\prime}\right] \subseteq \mathcal{D}_{2}\left(G^{\prime}\right)
$$


Finalizamos este item provando um importante resultado que estabelece uma $K$-base ordenada para álgebras $K G$ e particularmente descreve $K$-bases para os quocientes de potências do radical, ou melhor, explicita as $K^{*}$-bases para fatores da filtração de $I_{K^{*}}(G)$ :

$$
K G=I_{K^{*}}^{0}(G) \supseteq I_{K^{(}}^{1}(G) \supseteq \cdots \supseteq I_{K^{(}}^{k}(G) \supseteq I_{K^{*}}^{k+1}(G) \supseteq \cdots \supseteq I_{K^{*}}^{t}(G)=0,
$$

onde $t=t(G)$ é o índice de nilpotência de $I_{K}(G)$.

O seguinte lema tem muita importância, pois ele simplifica a dificuldade natural que aparece no artigo de Jennings.

Lema 2.4.5 Seja G o p-grupo não abeliano dado pela apresentação

$$
G=\left\langle a, b \mid a^{p^{n}}=1, b^{p^{m}}=a^{p^{t}},{ }^{b} a=a^{r}\right\rangle
$$

onde $t \geq 0, r^{p^{m}} \equiv 1\left(\bmod p^{n}\right)$ e $p^{t}(r-1) \equiv 0 \bmod \left(p^{n}\right)$ e seja $K$ um corpo de característica $p>0$. Considere o conjunto

$$
L_{k}=\left\{(1-a)^{k_{1}}(1-b)^{k_{2}} \mid 0 \leq k_{1}<p^{n}, 0 \leq k_{2} \leq p^{m}, k_{1}+k_{2}=k\right\}
$$

e seja $w: I_{K}(G) \rightarrow I_{K}^{k}(G) / I_{K}^{k+1}(G)$ a projeção canônica. Então a imagem $w\left(L_{k}\right)$ do conjunto $L_{k}$ é uma base do quociente $I_{K}^{k}(G) / I_{K}^{k+1}(G)$ como espaço vetorial sobre $K$.

Demonstração: Faremos indução sobre $k$, para provar que a imagem do conjunto $\{(1-$ $\left.a)^{l}(1-b)^{k-l} \mid 0 \leq l \leq k\right\}$ pela projeção canônica é um sistema de geradores do quociente $I_{K}^{k}(G) / I_{K}^{k+1}(G)$ como espaço vetorial sobre o corpo $K$. Seja $g, g^{\prime} \in G$, a identidade $1-g g^{\prime}=(1-g)+\left(1-g^{\prime}\right)-(1-g)\left(1-g^{\prime}\right)$ implica que a imagem do conjunto $\{(1-a),(1-b)\}$ pela projeção canônica é um sistema de geradores do quociente $I_{K^{*}}(G) / I_{K^{*}}^{2}(G)$ como espaço vetorial sobre $K$. Suponhamos por hipótese de indução, que $w\left(L_{m}\right)$ com $m \leq k$ seja um 
sistema de geradores do espaço vetorial $I_{K^{*}}^{m}(G) / I_{K^{*}}^{m+1}(G)$ e vamos provar que as imagens dos produtos $u_{1} u_{2}$, com $u_{1} \in I_{K}(G)$ e $u_{2} \in I_{K}^{m}(G)$ geram o quociente $I_{K}^{m+1}(G) / I_{K^{*}}^{m+2}(G)$ como espaço vetorial sobre $K$. Podemos escrever:

$$
\begin{aligned}
& w\left(u_{1}\right) \equiv \alpha_{1}(1-a)+\alpha_{2}(1-b)\left(\bmod I_{K^{-}}^{2}(G)\right), \operatorname{com} \alpha_{1}, \alpha_{2} \in K, \\
& w\left(u_{2}\right) \equiv \sum_{l=0}^{m} \beta_{l}(1-a)^{l}(1-b)^{m-l}\left(\bmod I_{K^{m}}^{m+1}(g)\right), \operatorname{com} \beta_{l} \in K, 0 \leq l \leq m .
\end{aligned}
$$

Portanto, temos o produto

(**) $w\left(u_{1} u_{2}\right) \equiv \sum_{l=0}^{m} \alpha_{1} \beta_{l}(1-a)^{l+1}(1+b)^{m-l}+\alpha_{2} \beta_{l}(1+b)(1+a)^{l}(1+b)^{m-l}\left(\bmod I_{K}^{m+2}(G)\right)$.

Vamos considerar primeiro o caso em que $p>2$. Denotamos $c=(b, a)=b a b^{-1} a^{-1}=$ ${ }^{b} a a^{-1}=a^{r} a^{-1}=a^{r-1}$ o gerador do subgrupo comutador de $G, G^{\prime}$. Pelo Resultado 2.4.4, $c \in G^{\prime} \subseteq \mathcal{D}_{2}(G)$ implica que $(1-c) \in I_{K^{*}}^{2}(G)$ e calculamos

$$
\begin{aligned}
(1-b)(1-a) & =(1-a)-(b-b a)=(1-a)-(b-c a b) \\
& =(1-a)-(1-c a) b=(1-a)+(1-c a)(1-b)-(1-c a) .
\end{aligned}
$$

Usando a identidade

$$
1-g g^{\prime}=(1-g)+\left(1-g^{\prime}\right)-(1-g)\left(1-g^{\prime}\right), \quad \forall g, g^{\prime} \in G
$$

que será de uso frequente daqui em diante, temos

$$
\begin{aligned}
(1-b)(1-a)= & (1-a)+[(1-c)+(1-a)-(1-c)(1-a)](1-b) \\
& -[(1-c)+(1-a)-(1-c)(1-a)] \\
= & (1-a)+(1-c)(1-b)+(1-a)(1-b)-(1-c)(1-a)(1-b) \\
& -(1-c)-(1-a)+(1-c)(1-a)
\end{aligned}
$$




$$
\begin{aligned}
= & (1-c)(1-b)+(1-a)(1-b)-(1-c)(1-a)(1-b) \\
& -(1-c)+(1-c)(1-a) .
\end{aligned}
$$

Como os termos $(1-c)(1-b),(1-c)(1-a)(1-b)$ e $(1-c)(1-a) \in I_{K^{(}}^{3}(G)$ e $c=a^{r-1}$, podemos escrever que

$$
(1-b)(1-a) \equiv(1-a)(1-b)-(1-c)\left(\bmod I_{K^{*}}^{3}(G)\right)
$$

isto é,

$$
\text { (*) }(1-b)(1-a) \equiv(1-a)(1-b)-\left(1-a^{r-1}\right)\left(\bmod I_{K}^{3}(G)\right) \text {. }
$$

Além disso, $r-1$ não pode ser coprimo com a ordem do elemento $a \in G$, pois caso contrário $G^{\prime}=\langle a\rangle$ implicaria que $G / G^{\prime}$ seria cíclico e pelo Resultado 2.1.3, $G$ seria cíclico (contradição). Como a ordem do elemento $a \in G$ é $p^{n}$, concluímos que $p$ divide $r-1$, isto é, existe um inteiro $s$ tal que $r-1=p s$. Na congruência $\left({ }^{*}\right)$, obtemos:

$$
(1-b)(1-a) \equiv(1-a)(1-b)-\left(1-a^{p s}\right)\left(\bmod I_{K^{-}}^{3}(G)\right)
$$

então

$$
(1-b)(1-a) \equiv(1-a)(1-b)-\left(1-a^{s}\right)^{p}\left(\bmod I_{K}^{3}(G)\right)
$$

e como $p>2$, temos:

$$
(1-b)(1-a) \equiv(1-a)(1-b)\left(\bmod I_{K}^{3} \cdot(G)\right)
$$

donde

$$
(1-b)(1-a)^{l}(1-b)^{m-l} \equiv(1-a)^{l}(1-b)^{m-l+1}\left(\bmod I_{K^{*}}^{m+2}(G)\right) .
$$

Substituindo em $\left({ }^{* *}\right)$, obtemos

$$
w\left(u_{1} u_{2}\right) \equiv \sum \alpha_{1} \beta_{l}(1-a)^{l+1}(1-b)^{m-l}--\alpha_{2} \beta_{l}(1-a)^{l}(1-b)^{m-l+1}\left(\bmod I_{K}^{m+2}(G)\right),
$$

provando o lema para $p>2$. 
A seguir, considere $p=2$. Tal como no caso anterior, pode-se provar que $p=2$ divide $r-1$ e escrevemos $r-1=2 s$. Se $s$ for um número par então 4 divide $r-1$ e um simples cálculo mostra que

$$
c+1=\left(a^{r-1}+1\right)=\left(a^{4 s^{\prime}}+1\right)=\left(a^{s^{\prime}}+1\right)^{4} \in I_{K^{\prime}}^{3}(G),
$$

logo, também teremos que

$$
(1+b)(1+a) \equiv(1+a)(1+b)\left(\bmod I_{K}^{3}(G)\right)
$$

donde, fazendo as mesmas substituições que foram feitas no caso anterior na congruência $\left.{ }^{* *}\right)$ o lema fica também provado quando $p=2$ e $4 / r-1$.

Resta agora somente considerar o caso em que $s$ é um inteiro ímpar, isto é, $s=2 j+1$. Temos que

$$
c+1=a^{r-1}+1=a^{2 s}+1=a^{4 j+2}+1=a^{4 j} \cdot a^{2}+1
$$

e pela identidade (2.4.5),

$$
c+1=\left(a^{4 j}+1\right)\left(a^{2}+1\right)+\left(a^{4 j}+1\right)+\left(a^{2}+1\right),
$$

donde claramente obtemos que

$$
c+1 \equiv(a+1)^{2}\left(\bmod I_{K}^{3}(G)\right) .
$$

Substituindo esta congruência na congruência $(*)$, temos:

$$
(1+b)(1+a) \equiv(1+a)(1+b)+(1+a)^{2}\left(\bmod I_{K^{(}}^{3}(G)\right)
$$

e por um simples cálculo, provamos que

$$
(* * *) \begin{cases}\text { se } l \text { é par, temos: } & (1+b)(1+a)^{l} \equiv(1+a)^{l}(1+b)\left(\bmod I_{K^{\prime}}^{l+2}(G)\right), \\ \text { se } l \text { é ímpar, temos: } & (1+b)(1+a)^{l} \equiv(1+a)^{l}(1+b)+(1+a)^{l+1}\left(\bmod I_{K}^{l+2}(G)\right) .\end{cases}
$$


Usando estes últimos resultados na congruência (**) obtemos

$$
\begin{aligned}
(* *) w\left(u_{1} u_{2}\right) \equiv & \sum_{l=0}^{m} \alpha_{1} \beta_{l}(1+a)^{l+1}(1+b)+\sum_{l \text { par }}^{m} \alpha_{2} \beta_{l}(1+b)(1+a)^{l}(1+b)^{m-1} \\
& +\sum_{l \text { ímpar }}^{m} \alpha_{2} \beta_{l}(1+b)(1+a)^{l}(1+b)^{m-l}\left(\bmod I_{K}^{m+2}(G)\right) .
\end{aligned}
$$

Agora, substituindo o resultado de $\left({ }^{* *}\right)$, obtemos

$$
\begin{aligned}
w\left(u_{1} u_{2}\right) \equiv & \sum_{l=0}^{m} \alpha_{1} \beta_{l}(1+a)^{l+1}(1+b)^{m-l} \\
& +\sum_{l \mathrm{par}}^{m} \alpha_{2} \beta_{l}(1+a)^{l}(1+b)^{m-l+1} \\
& +\sum_{\text {límpar }}^{m} \alpha_{2} \beta_{l}\left[(1+a)^{l}(1+b)^{m-l+1}+(1+a)^{l+1}(1+b)^{m-l}\right]\left(\bmod I_{K}^{m+2}(G)\right)
\end{aligned}
$$

concluindo a prova de que as imagens dos produtos $u_{1} u_{2}$ geram o quociente $I_{K^{\ddagger}}^{m+1}(G) / I_{K}^{m+2}(G)$ como espaço vetorial sobre $K$.

Finalizando a prova do lema basta ver que o conjunto $w\left(L_{K}\right)$ é linearmente independente sobre $K$, pelo seguinte: se $\sum_{t \in L_{K}} \alpha_{t} t \equiv 0\left(\bmod I_{K^{\prime}}^{k+1}(G)\right)$, onde $\alpha_{t} \in K$, então podemos escrever $\sum_{t \in L_{k}} \alpha_{t} t=\sum_{\substack{s \in \cup L_{l} \\ l>k}} \beta_{s} s$, onde $\beta_{s} \in K$. Conseqüentemente, $\alpha_{t}=0$, $\forall t \in L_{K}$ porque

$$
L_{k} \subset L=\left\{(1-a)^{k_{1}}(1-b)^{k_{2}} \mid 0 \leq k_{1}<p^{n} \quad \text { e } \quad 0 \leq k_{2}<p^{m}\right\}=\bigcup_{k=0}^{\downarrow}{ }^{n}+p^{m}-2,
$$

onde $L$ é uma $K$-base de $K G$ sobre $K$ (fato evidente devido $|G|=|L|$ ).

Tendo em vista a construção das $K$-bases multiplicativas filtradas de álgebras de grupo que aparecerão no teorema principal no final do capítulo seguinte, introduzimos na base $L$, acima dada a seguinte ordenação: seja $\left(k_{1}, k_{2}\right):=(1-a)^{k_{1}}(1-b)^{k_{2}}$ e definamos a ordem $\left(k_{1}, k_{2}\right)<\left(k_{1}^{\prime}, k_{2}^{\prime}\right)$ se, e somente se, $k_{2}<k_{2}^{\prime}$, ou $k_{2}=k_{2}^{\prime}$ e $k_{1}<k_{1}^{\prime}$. Claramente esta ordem preserva a ordenação natural dos subconjuntos $L_{k}$ com respeito aos seus elementos

$$
L_{0}<L_{1}<L_{2}<\cdots<L_{k}<\cdots
$$


e em cada $L_{k}$ os elementos $(1-a)^{k_{1}}(1-b)^{k_{2}}$ estão em ordem inferior se possuem o expoente do termo $(1-b)$ menor, onde $L$ tem a seguinte ordenação: 
$K$-base ordenada $L$

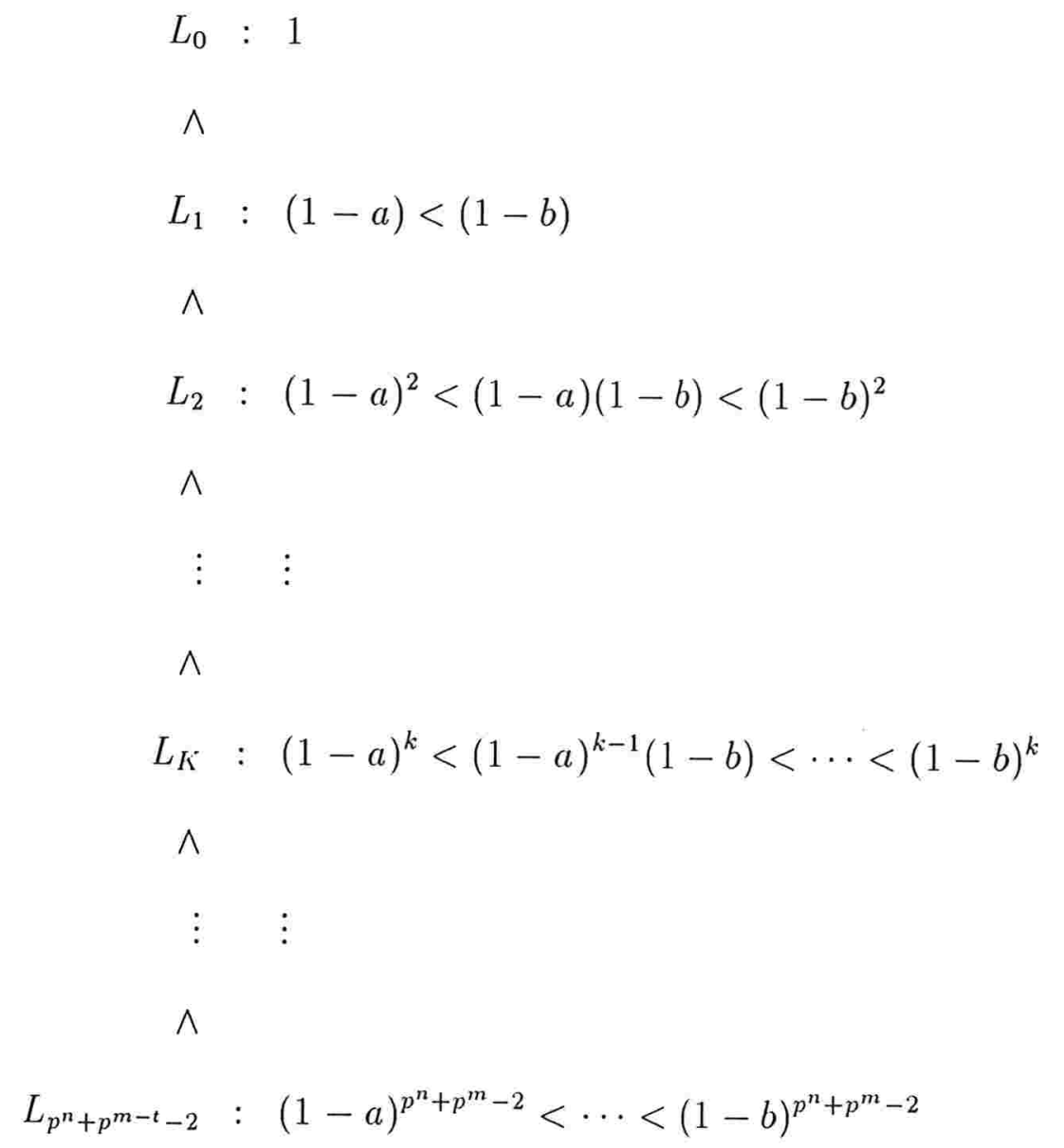

Usando o lema, determinamos as dimensões e as bases $w\left(L_{K^{*}}\right)$ dos quocientes $I_{K}^{k}(G) / I_{K}^{k+1}(G)$ como espaço vetorial sobre $K$, para os seguintes grupos. 
Nos seguintes exemplos considere $I=I_{K}(G)$ e $I^{0}=K^{\circ} G$.

Exemplo $1 G$ o grupo quatérnio de ordem oito, cuja apresentação é dada por $G=$ $\left\langle a, b \mid a^{4}=1, b^{2}=a^{2},{ }^{b} a=a^{-1}\right\rangle$.

\begin{tabular}{|c|c|c|}
\hline$K$-dimensão & Base $L_{k}$ & Quociente \\
\hline 1 & 1 & $I^{0} / I^{1}$ \\
\hline 2 & $(a+1) ; \quad(b+1)$ & $I / I^{2}$ \\
\hline 2 & $(a+1)^{2} ; \quad(a+1)(b+1)$ & $I^{2} / I^{3}$ \\
\hline 2 & $(a+1)^{3} ; \quad(a+1)^{2}(b+1)$ & $I^{3} / I^{4}$ \\
\hline 1 & $(a+1)^{3}(b+1)$ & $I^{4} / I^{5}$ \\
\hline
\end{tabular}


Neste trabalho interessarão grupos da forma

$$
G=\left\langle a, b \mid a^{2^{n}}=1, b^{2}=a^{2^{n-1}},{ }^{b} a=a^{-1+2^{n-1}}\right\rangle .
$$

Exemplo 2 Vamos calcular bases dos quocientes no caso em que $n=3$ do semi-diedral

$$
G=\left\langle a, b \mid a^{8}=1, b^{2}=a^{4},{ }^{b} a=a^{3}\right\rangle .
$$

\begin{tabular}{|c|cc|c|}
\hline$K$-dimensão & \multicolumn{2}{|c|}{ Base $L_{k}$} & Quocientes \\
\hline 1 & 1 & & $I^{0} / I^{1}$ \\
\hline 2 & $a+1$ & $b+1$ & $I / I^{2}$ \\
\hline 2 & $(a+1)^{2}$ & $(a+1)(b+1)$ & $I^{2} / I^{3}$ \\
\hline 2 & $(a+1)^{3}$ & $(a+1)^{2}(b+1)$ & $I^{3} / I^{4}$ \\
\hline 2 & $(a+1)^{4}$ & $(a+1)^{3}(b+1)$ & $I^{4} / I^{5}$ \\
\hline 2 & $(a+1)^{5}$ & $(a+1)^{4}(b+1)$ & $I^{5} / I^{6}$ \\
\hline 2 & $(a+1)^{6}$ & $(a+1)^{5}(b+1)$ & $I^{6} / I^{7}$ \\
\hline 2 & $(a+1)^{7}$ & $(a+1)^{6}(b+1)$ & $I^{7} / I^{8}$ \\
\hline 1 & $(a+1)^{7}(b+1)$ & $I^{8} / I^{9}$ \\
\hline
\end{tabular}


Exemplo 3 Consideremos ainda o caso em que $n=4$, do semi-diedral

$$
G_{4}=\left\langle a, b \mid a^{16}=1, b^{2}=a^{8},{ }^{b} a=a^{7}\right\rangle .
$$

\begin{tabular}{|c|ll|c|}
\hline K-dimensão & \multicolumn{2}{|c|}{$K$-base $L_{k}$} & Quociente \\
\hline 1 & 1 & $I^{0} / I^{1}$ \\
\hline 2 & $a+1$ & $b+1$ & $I / I^{2}$ \\
\hline 2 & $(a+1)^{2}$ & $(a+1)(b+1)$ & $I^{2} / I^{3}$ \\
\hline 2 & $(a+1)^{3}$ & $(a+1)^{2}(b+1)$ & $I^{3} / I^{4}$ \\
\hline 2 & $(a+1)^{4}$ & $(a+1)^{3}(b+1)$ & $I^{4} / I^{5}$ \\
\hline 2 & $(a+1)^{5}$ & $(a+1)^{4}(b+1)$ & $I^{5} / I^{6}$ \\
\hline 2 & $(a+1)^{6}$ & $(a+1)^{5}(b+1)$ & $I^{6} / I^{7}$ \\
\hline 2 & $(a+1)^{7}$ & $(a+1)^{6}(b+1)$ & $I^{7} / I^{8}$ \\
\hline 2 & $(a+1)^{8}$ & $(a+1)^{7}(b+1)$ & $I^{8} / I^{9}$ \\
\hline 2 & $(a+1)^{9}$ & $(a+1)^{8}(b+1)$ & $I^{9} / I^{10}$ \\
\hline 2 & $(a+1)^{10}$ & $(a+1)^{9}(b+1)$ & $I^{10} / I^{11}$ \\
\hline 2 & $(a+1)^{11}$ & $(a+1)^{10}(b+1)$ & $I^{11} / I^{12}$ \\
\hline 2 & $(a+1)^{12}$ & $(a+1)^{11}(b+1)$ & $I^{12} / I^{13}$ \\
\hline 2 & $(a+1)^{13}$ & $(a+1)^{12}(b+1)$ & $I^{13} / I^{14}$ \\
\hline 2 & $(a+1)^{14}$ & $(a+1)^{13}(b+1)$ & $I^{14} / I^{15}$ \\
\hline 2 & $(a+1)^{15}$ & $(a+1)^{14}(b+1)$ & $I^{15} / I^{16}$ \\
\hline 1 & $(a+1)^{15}(b+1)$ & $I^{16} / I^{17}$ \\
\hline
\end{tabular}




\section{Capítulo 3}

\section{Bases multiplicativas filtradas para p-grupos metacíclicos finitos}

\subsection{Introdução}

O principal objetivo deste capítulo é responder a questão proposta por R. Bautista, P. Gabriel, A. Roiter e L. Salmeron na referência [1], para a classe das álgebras de grupos modulares $K G$, onde $K$ é um corpo de característica $p>0$ e $G$ é um $p$-grupo metacíclico finito, com o seguinte teorema:

Teorema 3.1.1 Seja $G$ um p-grupo metacíclico não-abeliano finito e $K$ um corpo de característica $p>0$. Então a álgebra de grupo $K G$ admite uma base multiplicativa filtrada sobre o corpo $K$ se, e somente se, $p=2$ e apenas uma das seguintes condições vale:

1. G'é um grupo diedral; 
2. K contém uma raiz cúbica primitiva da unidade e $G$ é o grupo quatérnio de ordem 8 ;

Pelo acima descrito, o teorema principal descreve apenas os exemplos da álgebras de grupo com bases multiplicativas filtradas dadas por L. Paris [18].

Inicialmente apresentaremos dois lemas técnicos.

Lema 3.1.2 Seja G o 2-grupo semi-diedral de ordem $2^{n+1}$, cuja apresentação é dada por $G=\left\langle a, b \mid a^{2^{n}}=1, b^{2}=1,{ }^{b} a=a^{-1+2^{n-1}}\right\rangle$, com $n \geq 3$, e seja $K$ um corpo de característica 2. Considerando os elementos $u=a+b$ e $v=1+b$ da álgebra de grupo modular $K G$, com radical $I_{K}(G)$, então os seguintes itens valem:

(i) $\left(1+a^{-1}\right)=\sum_{i=1}^{2^{n}-1}(1+a)^{i}$ e $\left(1+a^{2^{n-1}-1}\right)=\sum_{i=1}^{2^{n-1}-1}(1+a)^{i}$;

(ii) $u^{2}=\sum_{i=2}^{2^{n-1}-1}(1+a)^{i}(1+b)+\sum_{j=3}^{2^{n-1}-1}(1+a)^{j}$;

(iii) $u^{2}-u v u=(1+a)^{2^{n-1}}(1+b)+(1+a)^{2^{n-1}} \neq 0$;

(iv) $u v u=\sum_{i=2}^{2^{n-1}-1}(1+a)^{i}(1+b)+\sum_{j=3}^{2^{n-1}-1}(1+a)^{j}$;

(v) $u^{2} \equiv u v u\left(\bmod I_{K}^{2^{n-1}}(G)\right)$, mas $u^{2}, u v u \notin I_{K^{*}}^{2^{n-1}}(G)$.

\section{Demonstração:}


(i) Seja $s=(1+a)+(1+a)^{2}+\cdots+(1+a)^{2^{n}-1}$. Então temos que

$$
(1+a) s=(1+a)^{2}+(1+a)^{3}+\cdots+(1+a)^{2^{n}},
$$

donde

$$
a s=s+(1+a) s=(1+a)+(1+a)^{2^{n}} .
$$

Como $(1+a)^{2^{n}}=0$, temos que

$$
s=\left(1+a^{-1}\right),
$$

donde segue a primeira igualdade.

Para a segunda igualdade deste item, tomamos $s=(1+a)+\cdots+(1+a)^{2^{n-1}-1} \mathrm{e}$ de modo análogo obtemos que $s=1+a^{2^{n-1}-1}$, que comprova a segunda igualdade.

(ii) Seja $r=-1+2^{n-1}$ e, pela apresentação de $G$, temos que $b^{2}=1$ e $c=(b, a)=$ ${ }^{b} a a^{-1}=a^{r-1}$ e $c a=a^{r}$. Então,

$$
\begin{aligned}
u^{2} & =(a+b)^{2}=[(1+a)+(1+b)]^{2} \\
& =(1+a)^{2}+(1+a)(1+b)+(1+b)(1+a)+(1+b)^{2} \\
& =(1+a)^{2}+(1+a)(1+b)+(1+b)(1+a) \\
& =(1+a)^{2}+(1+a)(1+b)+(1+a)+b(1+a) \\
& =(1+a)^{2}+(1+a)(1+b)+(1+a)+(b+b a) \\
& =(1+a)^{2}+(1+a)(1+b)+(1+a)+(b+c a b) \\
& =(1+a)^{2}+(1+a)(1+b)+(1+a)+(1+c a) b \\
& =(1+a)^{2}+(1+a)(1+b)+(1+a)+(1+c a)(1+b)+(1+c a) \\
& =(1+a)^{2}+(1+a)(1+b)+(1+a)+\left(1+a^{r}\right)(1+b)+\left(1+a^{r}\right)
\end{aligned}
$$

Substituindo da segunda igualdade já provada no item (i), obtemos que

$$
u^{2}=(1+a)^{2}+(1+a)(1+b)+(1+a)+\sum_{i=1}^{2^{n-1}-1}(1+a)^{i}(1+b)+\sum_{j=1}^{2^{n-1}-1}(1+a)^{j} .
$$


Simplificando termos, concluímos que

$$
u^{2}=\sum_{i=2}^{2^{n-1}-1}(1+a)^{i}(1+b)+\sum_{j=3}^{2^{n-1}-1}(1+a)^{j} .
$$

(iii) Sendo $v=1+b$, calculamos

$$
\begin{aligned}
u v u & =u(1+b) u=u^{2}+u b u=u^{2}+(a+b) b(a+b) \\
& =u^{2}+\left(a b+b^{2}\right)(a+b)=u^{2}+(a b+1)(a+b) \\
& =u^{2}+a b a+a+a b^{2}+b=u^{2}+a c a b+a+a+b \\
& =u^{2}+a a^{r} b+b=u^{2}+\left(a^{r+1}+1\right) b \\
& =u^{2}+\left(1+a^{r+1}\right)(1+b)+\left(1+a^{r+1}\right)
\end{aligned}
$$

e temos

$$
u^{2}-u v u=(1+a)^{2^{n-1}}(1+b)+(1+a)^{2^{n-1}} \neq 0 .
$$

(iv) Pelo item (iii), podemos escrever

$$
u v u=u^{2}+(1+a)^{2^{n-1}}(1+b)+(1+a)^{2^{n-1}}
$$

e usando o valor de $u^{2}$ dado no item (ii), temos

$$
u v u=\sum_{i=2}^{2^{n-1}-1}(1+a)^{i}(1+b)+\sum_{j=3}^{2^{n-1}-1}(1+a)^{j}+(1+a)^{2^{n-1}}(1+b)+(1+a)^{2^{n-1}},
$$

e concluímos que

$$
u v u=\sum_{i=2}^{2^{n-1}}(1+a)^{i}(1+b)+\sum_{j=3}^{2^{n-1}}(1+a)^{j}
$$

(v) Segue diretamente do item (iii), observando que $(1+a)^{2^{n-1}}(1+b)$ e $(1+a)^{2^{n-1}} \in$ $I_{K}^{2^{n-1}}(G)$, podemos escrever que $u^{2} \equiv u v u\left(\bmod I_{K}^{2^{n-1}}(G)\right)$ e pelos itens (ii) e (iv) claramente $u^{2}, u v u \notin I_{K}^{2^{n-1}}(G)$. 
Lema 3.1.3 Seja $G=\left\langle a, b \mid \cdot a^{2^{n}}=1, b^{2}=a^{2^{n-1}},{ }^{b} a=a^{-1}\right\rangle$, com $n>2$, e seja $K u$ um corpo de característica 2. Considere os elementos $u=a+b$ e $v=1+b$ da álgebra de grupo modular $K G$, com radical $I_{K}(G)$. Então temos que

(i) $(1+a)^{-1}=\sum_{i=1}^{2^{n}-1}(1+a)^{i}$;

(ii) $u^{2}=\sum_{i=2}^{2^{n}-1}(1+a)^{i}(1+b)+\sum_{j=3}^{2^{n}-1}(1+a)^{j}+(1+a)^{2^{n-1}}$;

(iii) $u^{2}-u v u=(1+a)^{2^{n-1}}(1+b)+(1+a)^{2^{n-1}} \neq 0$;

(iv) $u v u=\sum_{\substack{i=2 \\ i \neq 2^{n-1}}}^{2^{n}-1}(1+a)^{i}(1+b)+\sum_{j=3}^{2^{n}-1}(1+a)^{j}$

(v) $u^{2} \equiv u v u\left(\bmod I_{K}^{2^{n-1}}\left(G^{\prime}\right)\right)$ e $u^{2}, u v u \notin I_{K}^{2^{n-1}}(G)$.

\section{Demonstração:}

(i) Como $a^{2^{n}}=1$, repetimos o que foi feito no item (i) do lema anterior.

(ii) Pela apresentação dada, temos que $c={ }^{b} a a^{-1}=a^{-1} a^{-1}=a^{-2}$ e $c a=a^{-1}$, e escrevendo $r=-1$ temos que $b^{2}=a^{2^{n-1}}$ é um elemento do centro de $G$. Podemos também repetir o cálculo de $u^{2}$, substituindo no final $r=-1$. Assim obtemos:

$$
u^{2}=(1+a)^{2}+(1+a)(1+b)+(1+a)+(1+a)^{-1}(1+b)+\left(1+a^{-1}\right) .
$$

Substituindo conforme o item (i), temos

$$
u^{2}=(1+a)^{2}+(1+a)(1+b)+(1+a)+\sum_{i=1}^{2^{n}-1}(1+a)^{i}(1+b)+\sum_{j=1}^{2^{n}-1}(1+a)^{j} .
$$


Simplificando temos que

$$
u^{2}=\sum_{i=2}^{2^{n}-1}(1+a)^{i}(1+b)+\sum_{j=3}^{2^{n}-1}(1+a)^{j} .
$$

(iii) Sendo $v=1+b$, calculamos

$$
\begin{aligned}
u v u & =u(1+b) u=u^{2}+u b u=u^{2}+(a+b) b(a+b) \\
& =u^{2}+\left(a b+b^{2}\right)(a+b)=u^{2}+a b a+b^{2} a+b^{2} a+b^{3} .
\end{aligned}
$$

Sabemos que $b^{2}$ é central e estamos em característica 2 , logo,

$$
\begin{aligned}
u v u & =u^{2}+a b a+b^{3}=u^{2}+a c a b+b^{3}=u^{2}+a a^{-1} b+b^{3} \\
& =u^{2}+b+b^{3}=u^{2}+\left(1+b^{2}\right) b=u^{2}+\left(1+b^{2}\right)(1+b)+\left(1+b^{2}\right) .
\end{aligned}
$$

Sabemos que $b^{2}=a^{2^{n-1}}$ e substituindo acima, obtemos

$$
u v u=u^{2}+(1+a)^{2^{n-1}}(1+b)+(1+a)^{2^{n-1}},
$$

donde se conclui que

$$
u^{2}-u v u=(1+a)^{2^{n-1}}(1+b)+(1+a)^{2^{n-1}} \neq 0 .
$$

(iv) Pelo item (iii), podemos escrever

$$
u v u=u^{2}+(1+a)^{2^{n-1}}(1+b)+(1+a)^{2^{n-1}}
$$

e por $u^{2}$ dado em (ii),

$$
u v u=\sum_{i=2}^{2^{n}-1}(1+a)^{i}(1+b)+\sum_{j=3}^{2^{n}-1}(1+a)^{j}+(1+a)^{2^{n-1}}+(1+a)^{2^{n-1}}(1+b)+(1+a)^{2^{n-1}} .
$$

Simplificando termos, podemos concluir que

$$
u v u=\sum_{\substack{i=2 \\ i \neq 2^{n-1}}}^{2^{n}-1}(1+a)^{i}(1+b)+\sum_{j=3}^{2^{n}-1}(1+a)^{j} .
$$

(v) Diretamente do item (iii), observando que $(1+a)^{2^{n-1}}(1+b)$ e $(1+a)^{2^{n-1}} \in I_{K}^{2^{n-1}}(G)$, podemos escrever que $u^{2} \equiv u v u\left(\bmod I_{K}^{2^{n-1}}(G)\right)$ e pelos itens (ii) e (iv) claramente $u^{2}, u v u \notin I_{K}^{2^{n-1}}(G)$. 


\subsection{O teorema de classificação}

Antes de tratarmos do principal resultado deste trabalho, lembramos os seguintes fatos, importantes para o desenvolvimento da demonstração.

- Lembramos que ${ }^{b} a=b a b^{-1}$ e $(b, a)=b a b^{-1} a^{-1}={ }^{b} a a^{-1}$, notação introduzida no Resultado 2.1.2, página 8 .

- Vamos tratar exclusivamente dos p-grupos metacíclicos não abelianos finitos que pelo Resultado 2.1.1, tem apresentação dada por

$$
G=\left\langle a, b \mid a^{p^{n}}=1, b^{p^{m}}=a^{p^{t}},{ }^{b} a=a^{r}\right\rangle,
$$

onde $t \geq 0($ fixo $), r^{p^{m}} \equiv 1\left(\bmod p^{n}\right)$ e $p^{t}(r-1) \equiv 0\left(\bmod p^{n}\right)$.

- Todo elemento $g \in G$, supra citado, pode ser escrito como $g=a^{i} b^{j}$, onde $0 \leq i<p^{n}$ e $0 \leq j<p^{m}$.

- Faremos uso frequente da identidade (2.4.6)

$$
(x y-1)=(x-1)+(y-1)+(x-1)(y-1) .
$$

que apareceu no Lema 2.4.5.

- Sabemos que podemos obter todo elemento do radical $I_{K}(G)$ como uma combinação linear dos elementos da forma $(a-1)^{k}(b-1)^{l}$, onde $0 \leq k<p^{n}, 0 \leq l<p^{m}$ e $k+l \geq 1$.

O resultado principal a ser apresentado neste trabalho é o Teorema 3.1.1: 
Teorema Seja $G$ um p-grupo metacíclico não-abeliano finito e $K$ um corpo de característica $p>0$. Então a álgebra de grupo $K G$ admite uma base multiplicativa filtrada sobre o corpo $K$ se. e somente se, $p=2$ e uma das seguintes condições vale:

1. G é um grupo diedral;

2. $K$ contém uma raiz cúbica primitiva da unidade e $G$ é o grupo quatérnio de ordem 8 ;

Demonstração: Sabendo que $I_{K}(G)$ é o radical de $K G$ e $\operatorname{dim}_{K}(K G)=\operatorname{dim}_{K^{\prime}} I_{K^{-}}(G)+1$, suponha que $\{1\} \cup B$ é uma $K$-base multiplicativa filtrada de $K G$. Então, $B$ é uma $K$-base de $I_{K}(G)$. Obviamente $(a-1)^{i}(b-1)^{j} \in I_{K}^{2}(G)$ se $i+j \geq 2$ e $(a-1)$ e $(b-1)$ são geradores de $I_{K^{\prime}}(G)$ sobre $K$. Pelo Resutado 2.14, as classes $(a-1)+I_{K^{(}}^{2}(G)$ e $(b-1)+I_{K^{(}}^{2}(G)$ formam uma $K^{\prime}$-base do quociente $I_{K^{*}}(G) / I_{K^{\prime}}^{2}(G)$. Note que o subconjunto $B \backslash I_{K^{(}}^{2}(G)$, sabendo que pelo Resultado 2.2.5 que os elementos de $I_{K^{*}}^{2}(G)$ são não geradores da álgebra $I_{K^{*}}(G)$, consiste de dois elementos, que denotamos por $u$ e $v$. Podemos escrever a igualdade $K[u, v]=I_{K}(G)$, onde $u$ e $v$ são não comutativas. Além disso, temos que

$$
\begin{aligned}
& u \equiv \alpha_{1}(a-1)+\alpha_{2}(b-1)\left(\bmod I_{K^{(}}^{2}\left(G^{\prime}\right)\right) \\
& v \equiv \beta_{1}(a-1)+\beta_{2}(b-1)\left(\bmod I_{K^{\prime}}^{2}\left(G^{\prime}\right)\right)
\end{aligned}
$$

onde $\alpha_{i}, \beta_{i} \in K$ e $\Delta=\alpha_{1} \beta_{2}-\alpha_{2} \beta_{1} \neq 0$ (garantindo que $u, v$ são linearmente independentes). Do Resultado 2.4.4, $c=(b, a) \in G^{\prime} \subseteq \mathcal{D}_{2}(G)$, então $c-1 \in I_{K^{\prime}}^{2}(G)$ e por simples multiplicação obtemos

$$
\begin{aligned}
u v \equiv & {\left[\alpha_{1}(a-1)+\alpha_{2}(b-1)\right]\left[\beta_{1}(a-1)+\beta_{2}(b-1)\right]\left(\bmod I_{K^{-}}^{2}(G)\right) } \\
\equiv & \beta_{1} \alpha_{1}(a-1)^{2}+\beta_{1} \alpha_{2}(b-1)(a-1)+\beta_{2} \alpha_{1}(a-1)(b-1)+\beta_{2} \alpha_{2}(b-1)^{2} \\
& \left(\bmod I_{K^{-}}^{3}(G)\right) .
\end{aligned}
$$


Como

$$
\begin{aligned}
(b-1)(a-1)= & b(a-1)-(a-1)=(b a-b)-(a-1) \\
= & (c a b-b)-(a-1)=(c a-1) b-(a-1) \\
= & (c a-1)(b-1)+(c a-1)-(a-1) \\
= & (c-1)(a-1)(b-1)+(c-1)(b-1)+(a-1)(b-1) \\
& +(c-1)(a-1)+(c-1),
\end{aligned}
$$

substituindo em $u v$, obtemos

$$
\begin{aligned}
u v= & \beta_{1} \alpha_{1}(a-1)^{2}+\beta_{2} \alpha_{2}(b-a)^{2}+\left(\alpha_{1} \beta_{2}+\alpha_{2} \beta_{1}\right)(a-1)(b-1)+\alpha_{2} \beta_{1}(c-1) \\
& +\alpha_{2} \beta_{1}[(c-1)(a-1)(b-1)+(c-1)(b-1)+(c-1)(a-1)]
\end{aligned}
$$

e sabendo que $(c-1) \in I_{K^{-}}^{2}(G)$ e $(a-1),(b-1) \in I_{K}(G)$, temos que $(c-1)(a-1)(b-1)$, $(c-1)(b-1)$ e $(c-1)(a-1) \in I_{K}^{3}(G)$. Então, podemos escrever

$$
\begin{aligned}
u v= & \beta_{1} \alpha_{1}(a-1)^{2}+\beta_{2} \alpha_{2}(b-1)^{2}+\left(\alpha_{1} \beta_{2}+\alpha_{2} \beta_{1}\right)(a-1)(b-1) \\
& +\alpha_{2} \beta_{1}(c-1)\left(\bmod I_{K^{*}}^{3}(G)\right)
\end{aligned}
$$

Trabalhando de forma análoga, temos as seguintes congruências:

(2) $u v \equiv \alpha_{1} \beta_{1}(a-1)^{2}+\alpha_{2} \beta_{2}(b-1)^{2}+\left(\alpha_{1} \beta_{2}+\alpha_{2} \beta_{1}\right)(a-1)(b-1)+\alpha_{2} \beta_{1}(c-1)\left(\bmod I_{K^{-}}^{3}(G)\right)$;

(3) $v u \equiv \alpha_{1} \beta_{1}(a-1)^{2}+\alpha_{2} \beta_{2}(b-1)^{2}+\left(\alpha_{1} \beta_{2}+\alpha_{2} \beta_{1}\right)(a-1)(b-1)+\alpha_{1} \beta_{2}(c-1)\left(\bmod I_{K^{*}}^{3}(G)\right)$;

(4) $u^{2} \equiv \alpha_{1}^{2}(a-1)^{2}+\alpha_{2}^{2}(b-1)^{2}+2 \alpha_{1} \alpha_{2}(a-1)(b-1)+\alpha_{1} \alpha_{2}(c-1)\left(\bmod I_{K^{K}}^{3}(G)\right)$;

(5) $v^{2} \equiv \beta_{1}^{2}(a-1)^{2}+\beta_{2}^{2}(b-1)^{2}+2 \beta_{1} \beta_{2}(a-1)(b-1)+\beta_{1} \beta_{2}(c-1)\left(\bmod I_{K}^{3}(G)\right)$. 
Se $c-1 \in I_{K^{*}}^{3}(G)$, pelas congruências $(2)$ e $(3)$ obtemos que $u v \equiv v u\left(\bmod I_{K^{*}}^{3}(G)\right)$. Além disso, uv e vu não estão em $I_{K^{-}}^{3}(G)$ pois, caso contrário, sabendo que as projeçòes $(a-1)^{2},(b-1)^{2}$ e $(a-1)(b-1)$ formam uma $K^{\prime}$-base de $I_{K}^{2}(G) / I_{K}^{3}(G)$ e pelas relações dadas pelas congruências teríamos que $\alpha_{1} \beta_{1}=\alpha_{2} \beta_{2}=\alpha_{1} \beta_{2}+\alpha_{2} \beta_{1}=0$. Logo $\Delta=$ $\alpha_{1} \beta_{2}-\alpha_{2} \beta_{1}=-2 \alpha_{2} \beta_{1} \neq 0$ se, e somente se, $\alpha_{2} \neq 0$ e $\beta_{1} \neq 0$ (fora $p=2$ ) e isto implicaria que $\alpha_{1}=0$ e assim teríamos $\alpha_{2} \beta_{1}=0$ (contradição!), garantindo assim $u v, v u \notin I_{K^{\prime}}^{3}(G)$ e $u v \equiv v u\left(\bmod I_{K}^{3}(G)\right)$ que, pelo item (ii) da Proposição 2.3.1, concluímos que $u v=v u$, tornando a álgebra $I_{K}(G)$ comutativa, contradição!

Podemos assumir então que $c-1 \notin I_{K^{*}}^{3}(G)$. Pelo Resultado 2.1.3, para um $p$-grupo não abeliano o fator $G / G^{\prime}$ não pode ser cíclico. Afirmamos $(r-1,|a|) \neq 1$. De fato, pela apresentação de $G$ temos que $c=(b, a)={ }^{b} a a^{-1}=a^{r} a^{-1}=a^{r-1}$ e se $(r-1,|a|)=1$, ter-se-ia que $\langle a\rangle=\left\langle a^{r-1}\right\rangle=G^{\prime}$, então $G / G^{\prime}$ seria cíclico, contradição! Concluímos que $r-1$ é divisível por $p$ e assim escrevemos $r-1=p s$, para algum inteiro $s$. Voltando ao cálculo de $c-1$, temos que

$$
c-1=\left(a^{p s}-1\right)=\left(a^{s}-1\right)^{p} \in I_{K^{-}}^{3}(G),
$$

para $p>2$ e também se $p=2$ e $s$ par. Pelas observações acima, só existe a possibilidade que $s$ seja ímpar e $G$ é um 2-grupo com uma das seguintes apresentações:

(6) $G=\left\langle a, b \mid a^{2^{n}}=1, b^{2^{m}}=1,{ }^{b} a=a^{r}\right\rangle$, onde $r^{2^{m}} \equiv 1\left(\bmod 2^{n}\right)$ e $r \not \equiv 1(\bmod 4)$;

(7) $G=\left\langle a, b \mid a^{2^{n}}=1, b^{2^{m}}=a^{2^{n-1}},{ }^{b} a=a^{r}\right\rangle$, onde $r^{2^{m}} \equiv 1\left(\bmod 2^{n}\right), 2^{n-1}(r-1) \equiv$ $0\left(\bmod 2^{n}\right)$ e $r \not \equiv 1(\bmod 4)$.

Fazendo uma análise dos casos possíveis. temos os seguintes, que trataremos separadamente. 
Caso 1) Apresentação (6) com $m=1$ (ou $b^{2}=1$ ).

Caso 2) Apresentação (6) ou (7) com $m>1$.

Caso 3) Apresentação (7) $\operatorname{com} m=1$ e $n \geq 2$.

Caso 1. Suponhamos que $G$ tem apresentação (6) e $m=1$ (ou melhor $b^{2}=1$ ). Desde que $r-1=2 s$ e $s$ é ímpar, $s=2 k+1 \operatorname{com} k \geq 1$ e dado que $r^{2} \equiv 1\left(\bmod 2^{n}\right)$, tem para $r$ módulo $2^{n}$ as soluções $r=1, r=-1, r=1+2^{n-1}$ e $r=-1+2^{n-1}$ (vide referência [5, p.200], ou referência [15, p.56]) que $r=-1$ ou $r=-1+2^{n-1}$ (isto é, dois subcasos), $n \geq 3$, satisfazendo a condição $r \not \equiv 1(\bmod 4)$.

Usando a identidade (1), temos

$$
\begin{aligned}
c+1 & =a^{r-1}+1=a^{2 s}+1=a^{2(2 k+1)}+1=a^{4 k+2}+1=a^{4 k} a^{2}+1 \\
& =\left(a^{4 k}+1\right)\left(a^{2}+1\right)+\left(a^{4 k}+1\right)+\left(a^{2}+1\right)
\end{aligned}
$$

e, por indução, é fácil ver que $\left(a^{4 k}+1\right) \in I_{K^{(}}^{3}(G)$, que nos permite escrever

$$
c+1=\left(a^{s}+1\right)^{2} \equiv(a+1)^{2}\left(\bmod I_{K}^{3} \cdot(G)\right) .
$$

Levando este último resultado nas congruências (2) - (5), obtemos particularmente as congruências seguintes:

$$
\begin{cases}u v \equiv \beta_{1}\left(\alpha_{1}+\alpha_{2}\right)(1+a)^{2}+\Delta(1+a)(1+b) & \left(\bmod I_{K^{(}}^{3}(G)\right) \\ v u \equiv \alpha_{1}\left(\beta_{1}+\beta_{2}\right)(1+a)^{2}+\Delta(1+a)(1+b) & \left(\bmod I_{K^{(}}^{3}(G)\right) \\ u^{2} \equiv \alpha_{1}\left(\alpha_{1}+\alpha_{2}\right)(1+a)^{2} & \left(\bmod I_{K^{-}}^{3}(G)\right) \\ v^{2} \equiv \beta_{1}\left(\beta_{1}+\beta_{2}\right)(1+a)^{2} & \left(\bmod I_{K^{-}}^{3}(G)\right)\end{cases}
$$

Claramente, $u v, v u \notin I_{K^{(}}^{3}\left(G^{\prime}\right)$ e por $\Delta \neq 0$ implica que $u v \not \equiv v u\left(\bmod I_{K^{(}}^{3}(G)\right)$. E desde que a $K^{-}$-dimensão de $I_{K^{*}}^{2}\left(G^{\prime}\right) / I_{K^{*}}^{3}\left(G^{\prime}\right)$ é igual a 2 , o que é claro, pois os possíveis geradores 
módulo $I_{K^{-}}^{3}(G)$ pelo Lema 2.4.5 são $(a+1)^{2},(a+1)(b+1)$ e $(b+1)^{2}$. Mas $(b+1)^{2}=0$. Então os elementos $u v+I_{K^{(}}^{3}(G)$ e $v u+I_{K^{(}}^{3}(G)$ formam uma $K$-base de $I_{K^{*}}^{2}(G) / I_{K^{*}}^{3}(G)$, o que faz termos $u^{2}, v^{2} \in I_{K}^{3}(G)$. Logo, obtemos as igualdades

$$
\alpha_{1}\left(\alpha_{1}+\alpha_{2}\right)=0 \quad \text { e } \quad \beta_{1}\left(\beta_{1}+\beta_{2}\right)=0
$$

que nos garante as soluções

$$
u=\alpha(a+b) \quad \text { e } \quad v=\beta(1+b)
$$

$\operatorname{com} \alpha, \beta \in K \backslash\{0\}$. Podemos tomar $\alpha=\beta=1$ e então

$$
u=a+b \quad \text { e } \quad v=1+b .
$$

Mais precisamente, $u \equiv(a+b)+I_{K^{j}}^{2}(G)$ e $v=(1+b)+I_{K^{K}}^{2}(G)$.

Podemos agora estudar o subcaso 1.1, onde $r=-1$, que nos dá a apresentação

$$
G=\left\langle a, b \mid a^{2^{n}}=1, b^{2}=1,{ }^{b} a=a^{-1}\right\rangle,
$$

com $n \geq 2$, que é o 2-grupo diedral de ordem $2^{n+1}$.

Provaremos independentemente na Proposição 3.1.2 que a álgebra de grupo do grupo diedral de ordem $2^{n+1}$ admite base multiplicativa filtrada.

Podemos agora estudar o subcaso 1.2 , onde $s=r=-1+2^{n-1}, n \geq 3$, que nos dá a apresentação

$$
G=\left\langle a, b \mid a^{2 n}=1, b^{2}=1,{ }^{b} a=a^{-1+2^{n-1}}\right\rangle, \quad \text { com } n \geq 3,
$$

conhecido como o grupo semidiedral de ordem $2^{n+1}$, onde vamos agora provar que a álgebra de grupo nào possui uma $K$-base multiplicativa filtrada pelo seguinte: se a 
álgebra de grupo modular para o grupo semi-diedral tem uma $K$-base multiplicativa filtrada $B \operatorname{com} u, v \in B$, então considere o item (v) do Lema 3.1.2, página 27 , onde $u^{2} \equiv$ $u v u\left(\bmod I_{K}^{2^{n-1}}(G)\right)$, com $u^{2}, u v u \notin I_{K}^{2^{n-1}}(G)$. Nas hipóteses do item (ii) da Proposição 2.3.1 concluímos que $u^{2}=u v u$, mas pelo item (iii) do Lema 3.1.2 temos $u^{2}-u v u=$ $(1+a)^{2^{n-1}}(1+b)+(1+a)^{2^{n-1}} \neq 0$, contradição.

Caso 2. Estudamos agora o caso em que o grupo $G$ tem as apresentações (6) com $m>1$ ou (7) $\operatorname{com} m>1$. Isto é,

(6) $G=\left\langle a, b \mid a^{2^{n}}=1, b^{2^{m}}=1,{ }^{b} a=a^{r}\right\rangle$, onde $r^{2^{m}} \equiv 1\left(\bmod 2^{n}\right)$ e $r \not \equiv 1(\bmod 4)$;

(7) $G=\left\langle a, b \mid a^{2^{n}}=1, b^{2^{m}}=a^{2^{n-1}},{ }^{b} a=a^{r}\right\rangle$, onde $r^{2^{m}} \equiv 1\left(\bmod 2^{n}\right), 2^{n-1}(r-1) \equiv$ $0\left(\bmod 2^{n}\right)$ e $r \not \equiv 1(\bmod 4)$.

Observação: Os grupos de (6) e (7) são isomorfos (fazer $\left.b^{\prime}=a b \mathrm{em}(7)\right)$.

Calculamos

$$
\begin{aligned}
(1+b)(1+a) & =(1+a)+b(1+a)=(1+a)+(b+b a)=(1+a)+(b+c a b) \\
& =(1+a)+(1+c a) b=(1+a)+(1+c a)(1+b)+(1+c a) .
\end{aligned}
$$

Usando a identidade 2.4.6, temos que

$$
\begin{aligned}
(1+b)(1+a)= & (1+a)+[(1+c)(1+a)+(1+c)+(1+a)](1+b)+(1+c)(1+a) \\
& +(1+c)+(1+a) \\
= & (1+a)(1+b)+(1+c)+(1+c)(1+a)(1+b)+(1+c)(1+b) \\
& +(1+c)(1+a),
\end{aligned}
$$


e sabendo que $(1+c) \in I_{K}^{2}(G)$ e $(1+c) \equiv(1+a)^{2}\left(\bmod I_{K}^{3}(G)\right)$, podemos concluir que

$$
(1+b)(1+a) \equiv(1+a)(1+b)+(1+a)^{2}\left(\bmod I_{K}^{3}(G)\right) .
$$

Introduzindo esta relação nas congruências (2) - (5), obtemos neste caso particular

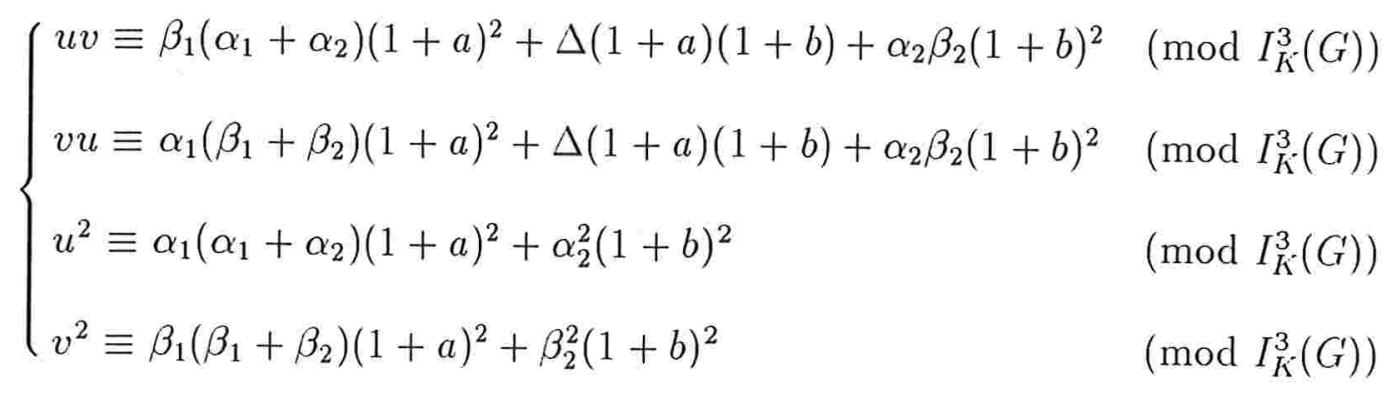

Sendo $\Delta \neq 0$, obtemos que $u v, v u \notin I_{K^{\prime}}^{3}\left(G^{\prime}\right)$ e $u v \not \equiv v u\left(\bmod I_{K^{*}}^{3}(G)\right)$, pois caso contrário teríamos $\beta_{1}\left(\alpha_{1}+\alpha_{2}\right)=\alpha_{1}\left(\beta_{1}+\beta_{2}\right)$, o que implicaria $\beta_{1} \alpha_{1}+\beta_{1} \alpha_{2}=\alpha_{1} \beta_{1}+\alpha_{1} \beta_{2}$, e então $\beta_{1} \alpha_{2}=\alpha_{1} \beta_{2}$, que faz $\Delta=0$ (contradição!) Além disso, $u^{2}+I_{K}^{3}(G)$ e $v^{2}+I_{K}^{3}(G)$ devem ser elementos não nulos do quociente $I_{K}^{2}(G) / I_{K}^{3}(G)$, pois se $\alpha_{1}\left(\alpha_{1}+\alpha_{2}\right)=0$ e $\alpha_{2}^{2}=0$, entào $\alpha_{1}=\alpha_{2}=0$ e $\operatorname{logo} \Delta=0$ (contradição) e se $\beta_{1}\left(\beta_{1}+\beta_{2}\right)=0$ e $\beta_{2}^{2}=0$, então $\beta_{1}=\beta_{2}=0$ e logo $\Delta=0$ (contradição).

É imediato ver que $u v \not \equiv v^{2}$. $v u \not \equiv v^{2}$ e $u v \not \equiv u^{2}, v u \not \equiv u^{2}$.

Afirmamos que a $K^{\prime}$-dimensão do quociente $I_{K^{-}}^{2}(G) / I_{K^{\prime}}^{3}(G)$ é 3. De fato, pelo Lema 2.4.5, temos que os elementos de uma $K^{\prime}$-base, módulo $I_{K}^{3}(G)$, são os seguintes:

$$
(a+1)^{2} ;(a+1)(b+1) \text { e }(b+1)^{2} .
$$

Para que os quatro elementos de (9) formem uma base do quociente a única possibilidade é que $u^{2} \equiv v^{2}\left(\bmod I_{K^{3}}^{3}(G)\right)$. Pelo item (ii) da Proposição 2.3.1, neste caso temos que $u^{2}=v^{2}$. de onde obtemos $\alpha_{2}^{2}=\beta_{2}^{2}$ e $\alpha_{1}\left(\alpha_{1}+\alpha_{2}\right)=\beta_{1}\left(\beta_{1}+\beta_{2}\right)$. Logo, $\alpha_{2}=\beta_{2}$ e ambos devem ser diferentes de zero, pois em caso contrário, ter-se-ia que $\Delta=0$. A segunda 
igualdade mostra que considerando esta expressão como uma equação do segundo grau em $\alpha_{1}$, temos que $\alpha_{1}=\beta_{1}+\beta_{2}$ ou $\alpha_{1}=\beta_{1}+\beta_{2}$ e

$$
u=(1+\lambda) a+b+\lambda \quad \text { e } \quad v=\lambda a+b+\lambda+1
$$

$\operatorname{com} \lambda=\frac{\beta_{1}}{\beta_{2}}$ e $\Delta=\beta_{2}^{2} \neq 0$. Então, na igualdade $v^{2}=u^{2}$, obtemos a relação $\left(1+a^{2}\right)+$ $a b+b a=0$. Isto é uma contradição com as relações dadas nas apresentações (6) e (7) do grupo $G$, pois esta relação só é verdadeira se $1=a^{2}$ e $a b=b a$ ou $1=a b$ e $a^{2}=b a$ ou $1=b a$ e $a^{2}=a b$ e todas estas fazem $G$ abeliano (contradição com a hipótese do Teorema!). Conseqüentemente, nestas condições a álgebra de grupo $K G$ (não comutativa) não admite uma $K$-base multiplicativa filtrada.

Caso 3. Neste caso $G$ tem a apresentação (7), $m=1$ e $n \geq 2$. Do estudo do Caso 1 , já sabemos que quando $m=1$ só é possível termos $r=-1$ ou $r=-1+2^{n-1}$ e, assim, vamos dividir o nosso estudo em quatro subcasos que denominaremos por $3.1,3.2,3.3$ e 3.4 .

Subcaso 3.1. Tomamos $n=2, r=-1$ e neste caso $G$ tem apresentação

$$
G=\left\langle a, b \mid a^{4}=1, b^{2}=a^{2},{ }^{b} a=a^{-1}\right\rangle .
$$

que é o conhecido grupo quatérnios de ordem oito.

Mostraremos separadamente na Proposição 3.1.3 que a álgebra de grupo do quatérnio de ordem oito admite base multiplicativa filtrada se e somente se o corpo $K$ contém uma raiz cúbica primitiva da unidade.

Subcaso 3.2. Consideramos $n>2$ e $r=-1$ e obtemos a apresentação

$$
G=\left\langle a, b \mid a^{2^{n}}=1, b^{2}=a^{2^{n-1}},{ }^{b} a=a^{-1}\right\rangle .
$$


Agora, $G$ é o grupo quatérnio generalizado e a álgebra de grupo modular $K G$ não tem uma base multiplicativa filtrada pelo seguinte (análogo ao que foi feito para a álgebra de grupo do grupo semidiedral): se a álgebra de grupo modular para o grupo quatérnio generalizado tem uma $K$-base multiplicativa filtrada $B \operatorname{com} u, v \in B$, então considere o item (v) do Lema 3.1.2, página 27 , onde $u^{2} \equiv u v u\left(\bmod I_{K^{2}}^{2^{n-1}}(G)\right), \operatorname{com} u^{2}, u v u \notin I_{K^{*}}^{2^{n-1}}(G)$ que nas hipóteses do item (ii) da Proposição 2.3.1 concluímos que $u^{2}=u v u$, mas pelo item (iii) do Lema 3.1.2 temos $u^{2}-u v u=(1+a)^{2^{n-1}}(1+b)+(1+a)^{2^{n-1}} \neq 0$, contradição.

Concluímos que para a álgebra de grupo modular do quatérnio generalizado não existe $K$-base multiplicativa filtrada.

Subcaso 3.3. Consideramos $G$ na apresentação (7), com $n=2$ e $r=-1+2^{n-1}$. Então, $r=1 \mathrm{e}$

$$
G=\left\langle a, b \mid a^{4}=1, b^{2}=a^{2},{ }^{b} a=a\right\rangle,
$$

onde claramente $a$ e $b$ comutam e $G$ é grupo abeliano (contradição).

Subcaso 3.4. Para concluirmos resta o estudo do subcaso 3.4, onde $G$ tem a apresentação (7), com $n>2$ e $r=-1+2^{n-1}$. Agora temos

$$
G=\left\langle a, b \mid a^{2^{n}}=1, b^{2}=a^{2^{n-1}},{ }^{b} a=a^{-1+2^{n-1}}\right\rangle,
$$

que é um grupo isomorfo ao grupo semi-diedral já tratado.

Mostraremos separadamente na Observação 3.1.4 que o grupo semi-diedral possui uma base filtrada, mas não multiplicativa, i.e, $B \cup\{0\}$ para multiplicação da álgebra não é um semi-grupo. 
Vamos agora tratar separadamente das construções das $K$-bases multiplicativas filtradas para as álgebras de grupos modulares dos 2-grupos diedral, quatérnio de ordem 8 e pelas seguintes proposições:

Proposição 3.2.1 Seja $G=\left\langle a, b \mid a^{2^{n}}=1, b^{2}=1,{ }^{b} a=a^{-1}\right\rangle$ o 2-grupo diedral de ordem $2^{n+1}$ e K um corpo de característica 2, então o conjunto

$$
B=\left\{1, v, u^{i}, v u^{i}, u^{j} v, v u^{j} v \mid i=1, \ldots, 2^{n-1} \text { e } j=1, \ldots, 2^{n-1}-1\right\}
$$

onde $u=a+b$ e $v=1+b$ é uma $K$-base multiplicativa filtrada de $K G$.

(A base que construímos nesta proposição aparece no artigo de L. Paris [18] porém sem justificativa alguma.)

Demonstração: Inicialmente, vamos provar a seguinte afirmação:

Afirmação: Nas condições acima vale:

(*) $u^{i} \equiv(1+a)^{2 i-1}+(1+a)^{2 i-2}(1+b)+\gamma_{i}\left[(1+a)^{2 i}+(1+a)^{2 i-1}(1+b)\right]\left(\bmod I_{K}^{2 i+1}(G)\right)$, onde $\gamma \equiv(1+i)(\bmod 2)$.

Vamos provar a afirmação por indução em $i$. Para $i=1(\gamma=0)$, temos pela fórmula $(*)$

$$
u=u^{1} \equiv(1+a)^{1}+(1+a)^{0}(1+b)+0\left[(1+a)^{2}+(1+a)^{1}(1+b)\right]\left(\bmod I_{K^{-}}^{3}(G)\right) .
$$

Portanto, $u \equiv(a+b)\left(\bmod I_{K}^{3}(G)\right)$, o que é verdadeiro. 
Para $i=2(\gamma=1)$. Temos pela fórmula $\left({ }^{*}\right)$

$$
u^{2} \equiv(1+a)^{3}+(1+a)^{2}(1+b)+(1+a)^{4}+(1+a)^{3}(1+b)\left(\bmod I_{K^{(}}^{5}(G)\right)
$$

e por um simples cálculo

$$
\begin{aligned}
u^{2} & =(a+b)^{2}=[(1+a)+(1+b)]^{2} \\
& =(1+a)^{2}+(1+a)(1+b)+(1+b)(1+a)+(1+b)^{2} \\
& =(1+a)^{2}+(1+a)(1+b)+(1+b)(1+a),
\end{aligned}
$$

pois $b^{2}=1$. Pelo cálculo já feito na página 39 , temos que

$$
\begin{aligned}
(1+b)(1+a) & =(1+a)+(1+c a)(1+b)+(1+c a) \\
& =(1+a)+\left(1+a^{-1}\right)(1+b)+\left(1+a^{-1}\right)
\end{aligned}
$$

e pela igualdade $\left(1+a^{-1}\right)=\sum_{j=1}^{2^{n}-1}(1+a)^{j}$ (já provada na página 28$)$, podemos escrever que

$$
\begin{aligned}
(1+b)(1+a) \equiv & (1+a)(1+b)+(1+a)^{2}(1+b)+(1+a)^{3}(1+b) \\
& +(1+a)^{2}+(1+a)^{3}+(1+a)^{4}\left(\bmod I_{K^{-}}^{5}(G)\right)
\end{aligned}
$$

que substituindo no cálculo de $u^{2}$ concluímos

$$
u^{2} \equiv(1+a)^{3}+(1+a)^{2}(1+b)+(1+a)^{4}+(1+a)^{3}(1+b)\left(\bmod I_{K^{*}}^{5}(G)\right)
$$

e, assim, ficou provada a validade de $u^{i}$, para $i=1,2$.

Considere por hipótese de indução que a fórmula $(*)$ vale para $i$ e vamos provar que vale para $i+1$, ou melhor, provaremos que

$$
\begin{aligned}
u^{i+1} \equiv & (1+a)^{2 i+1}+(1+a)^{2 i}(1+b)+(1+\gamma)\left[(1+a)^{2 i+2}\right. \\
& \left.+(1+a)^{2 i+1}(1+b)\right]\left(\bmod I_{K}^{2 i+3}(G)\right)
\end{aligned}
$$


Temos

$$
\begin{aligned}
u^{i+1}= & u^{i} u=u^{i}[(1+a)+(1+b)] \\
\equiv & {\left[(1+a)^{2 i-1}+(1+a)^{2 i-2}(1+b)+\gamma(1+a)^{2 i}\right.} \\
& \left.+\gamma(1+a)^{2 i-1}(1+b)\right][(1+a)+(1+b)]\left(\bmod I_{K}^{2 i+3}(G)\right) \\
\equiv & (1+a)^{2 i}+(1+a)^{2 i-2}(1+b)(1+a)+\gamma(1+a)^{2 i+1}+\gamma(1+a)^{2 i-1}(1+b)(1+a) \\
& +(1+a)^{2 i-1}(1+b)+(1+a)^{2 i-2}(1+b)^{2}+\gamma(1+a)^{2 i}(1+b) \\
& +\gamma(1+a)^{2 i-1}(1+b)^{2}\left(\bmod I_{K}^{2 i+3}(G)\right) \\
\equiv & (1+a)^{2 i}+(1+a)^{2 i-2}(1+b)(1+a)+\gamma(1+a)^{2 i+1}+\gamma(1+a)^{2 i-1}(1+b)(1+a) \\
& +(1+a)^{2 i-1}(1+b)+\gamma(1+a)^{2 i}(1+b)\left(\bmod I_{K}^{2 i+3}(G)\right) .
\end{aligned}
$$

Calculando separadamente,

$$
\begin{aligned}
(1+b)(1+a) & =(1+a)+b(1+a)=(1+a)+(b+b a) \\
& =(1+a)+(b+c a b)=(1+a)+(1+c a)(1+b)+(1+c a)
\end{aligned}
$$

e pela apresentação do grupo diedral, temos que $c a=b a b^{-1} a^{-1} a={ }^{b} a=a^{-1}, \operatorname{logo}$

$$
(1+b)(1+a)=(1+a)+(1+a)+\left(1+a^{-1}\right)(1+b)+\left(1+a^{-1}\right)
$$

e pelo item (i) do Lema 3.1.2 tem-se que $\left(1+a^{-1}\right)=\sum_{i=1}^{2^{n}-1}(1+a)^{i}$ donde obtemos que

$$
\begin{aligned}
(1+b)(1+a) & =(1+a)+\sum_{i=1}^{2^{n}-1}(1+a)^{i}(1+b)+\sum_{j=1}^{2^{n}-1}(1+a)^{j} \\
& =\sum_{j=1}^{2^{n}-1}(1+a)^{i}(1+b)+\sum_{j=2}^{2^{n}-1}(1+a)^{j}
\end{aligned}
$$

levando este último resultado a expressão de $u^{i+1}$ podemos continuar o cálculo da congruência:

$$
u^{i+1} \equiv(1+a)^{2 i}+(1+a)^{2 i-2}\left[\sum_{j=1}^{2^{n}-1}(1+a)^{j}(1+b)+\sum_{j=2}^{2^{n}-1}(1+a)^{j}\right]
$$




$$
\begin{aligned}
& +\gamma(1+a)^{2 i+1}+\gamma(1+a)^{2 i}\left[\sum_{j=1}^{2^{n}-1}(1+a)^{i}(1+b)+\sum_{j=2}^{2^{n}-1}(1+a)^{j}\right] \\
& +(1+a)^{2 i-1}(1+b)+\gamma(1+a)^{2 i}(1+b) \\
\equiv & (1+a)^{2 i}+(1+a)^{2 i-1}(1+b)+(1+a)^{2 i}(1+b)+(1+a)^{2 i+1}(1+b) \\
& +(1+a)^{2 i}+(1+a)^{2 i+1}+(1+a)^{2 i+2}+\gamma(1+a)^{2 i+1}+\gamma(1+a)^{2 i}(1+b) \\
& +\gamma(1+a) 2 i+1(1+b)+\gamma(1+a)^{2 i+1}+\gamma(1+a)^{2 i+2}+(1+a)^{2 i-1}(1+b) \\
& +\gamma(1+a)^{2 i}(1+b)\left(\bmod I_{K}^{2 i+3}(G)\right),
\end{aligned}
$$

Simplificando e reagrupando adequadamente os termos,

$u^{i+1} \equiv(1+a)^{2 i+1}+(1+a)^{2 i}(1+b)+(1+\gamma)\left[(1+a)^{2 i+2}+(1+a)^{2 i+1}(1+b)\right]\left(\bmod I_{K}^{2 i+3}(G)\right)$. e vale nossa afirmação.

Fazendo uso da fórmula $\left(^{*}\right)$ para $u^{i}$, obtemos as seguintes congruências:

$$
\begin{aligned}
u^{i} & \equiv(1+a)^{2 i-1}+(1+a)^{2 i-2}(1+b)\left(\bmod I_{K^{K}}^{2 i}(G)\right), \\
v u^{i} & \equiv(1+a)^{2 i}+(1+a)^{2 i-1}(1+b)\left(\bmod I_{K}^{2 i+1}(G)\right), \\
u^{j} v & \equiv(1+a)^{2 j-1}(1+b)\left(\bmod I_{K^{i}}^{2 j+1}(G)\right), \\
v u^{j} v & \equiv(1+a)^{2 j}(1+b)\left(\bmod I_{K^{j}}^{2 j+2}(G)\right),
\end{aligned}
$$

para $i=1,2, \ldots, 2^{n-1}$ e $j=1,2, \ldots, 2^{n-1}-1$.

Pelo Lema 2.4.5 e sabendo que $(1+b)^{2}=0$, observa-se que o quociente $I_{K^{-}}^{t}(G) / I_{K^{*}}^{t+1}(G)$ tem a seguinte $K$-base:

$$
(a+1)^{t}+I_{K^{\prime}}^{t+1}(G) \text { e }(a+1)^{t-1}(b+1)+I_{K}^{t+1}(G)
$$

considerando $t=2 k+1$, onde $k=1,2, \ldots, 2^{n-2}-1$. E, assim, temos

$$
u^{k+1} \equiv(1+a)^{2 k+1}+(1+a)^{2 k}(1+b)\left(\bmod I_{K}^{2 k+2}(G)\right),
$$




$$
v u^{k} v \equiv(1+a)^{2 k}+(1+b)\left(\bmod I_{K}^{2 k+2}(G)\right),
$$

e claramente $u^{k+1}$ e $v u^{k} v$ são linearmente independentes módulo $I_{K^{k}}^{2 k+2}(G)$. Também para $t=2 k$, onde $k=1, \ldots, 2^{n-2}-1$, obtemos que

$$
v u^{k} \equiv(1+a)^{2 k}+(1+a)^{2 k-1}(1+b)\left(\bmod I_{K}^{2 k+2}(G)\right)
$$

e

$$
u^{k} v \equiv(1+a)^{2 k-1}(1+b)\left(\bmod I_{K}^{2 k+2}(G)\right)
$$

e, portanto, $v u^{k}$ e $u^{k} v$ são linearmente independentes módulo $I_{K^{2}}^{2 k+2}(G)$. A matriz dos coeficientes destes elementos, escritos em função da $K$-base ordenada $L$ é:

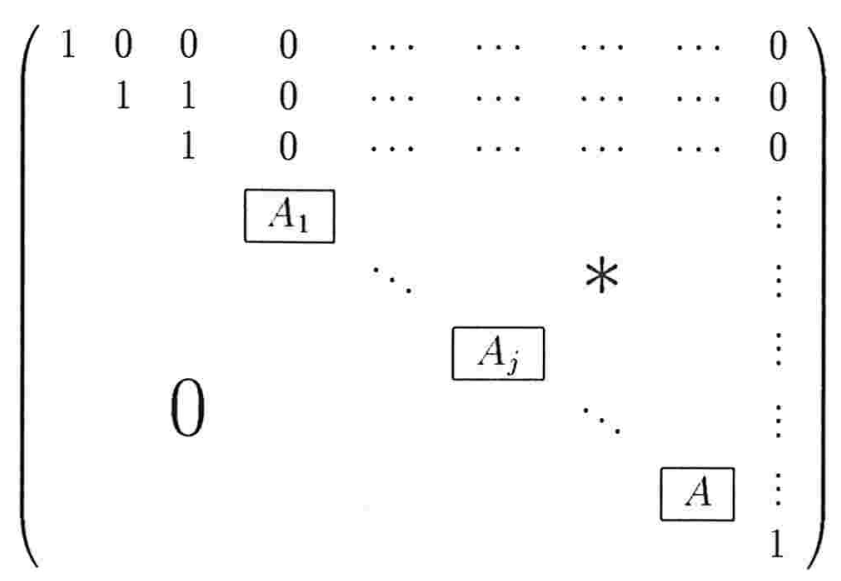

onde $A_{j}=\left[\begin{array}{ll}1 & 1 \\ 0 & 1\end{array}\right], \operatorname{com} j=1, \ldots, 2^{n}-1$, é unimodular. Desta forma o conjunto

$$
\left\{1, v, u^{i}, v u^{i}, u^{j} v, v u^{j} v \mid i=1, \ldots, 2^{n-1} \text { e } \quad j=1, \ldots, 2^{n-1}-1\right\}
$$

forma uma $K$-base multiplicativa filtrada de $K G$, como queríamos demonstrar.

Vamos construir uma $K$-base multiplicativa filtrada para álgebra de grupo modular do grupo quatérnio de ordem oito provando a seguinte proposição: 
Proposição 3.2.2 Seja $G=\left\langle a, b \mid a^{4}=1, b^{2}=a^{2},{ }^{b} a=a^{-1}\right\rangle$ e $K$ um corpo de característica 2. Então a álgebra de grupo $K G$ tem uma $K$-base multiplicativa filtrada $B$, com $u, v \in B$, se e somente se o corpo İ contém uma raiz cúbica primitiva da unidade.

Demonstração: Considere as congruências (2) - (5) dadas na página 35, particularmente para o grupo quatérnio, de onde obtemos as congruências

$$
(q) \begin{cases}u v \equiv\left(\alpha_{1} \beta_{1}+\alpha_{2} \beta_{1}+\alpha_{2} \beta_{2}\right)(1+a)^{2}+\Delta(1+a)(1+b) & \left(\bmod I_{K}^{3}(G)\right) \\ v u \equiv\left(\alpha_{1} \beta_{1}+\alpha_{1} \beta_{2}+\alpha_{2} \beta_{2}\right)(1+a)^{2}+\Delta(1+a)(1+b) & \left(\bmod I_{K}^{3}(G)\right) \\ u^{2} \equiv\left(\alpha_{1}^{2}+\alpha_{1} \alpha_{2}+\alpha_{2}^{2}\right)(1+a)^{2} & \left(\bmod I_{K}^{3}(G)\right) \\ v^{2} \equiv\left(\beta_{1}^{2}+\beta_{1} \beta_{2}+\beta_{2}^{2}\right)(1+a)^{2} & \left(\bmod I_{K}^{3}(G)\right)\end{cases}
$$

Como $\Delta \neq 0$, temos que $u v, v u \notin I_{K^{*}}^{3}(G)$ e se $u v \equiv v u\left(\bmod I_{K^{*}}^{3}(G)\right)$ ter-se-ia que $\alpha_{1} \beta_{1}+$ $\alpha_{2} \beta_{1}+\alpha_{2} \beta_{2}=\alpha_{1} \beta_{1}+\alpha_{1} \beta_{2}+\alpha_{2} \beta_{2}$ que faz $\lrcorner=0$, contradição! Logo, $u v \not \equiv v u\left(\bmod I_{K^{(}}^{3}(G)\right)$ e, além disso, é evidente que $u v \not \equiv v^{2}, v u \not \equiv v^{2}$ e $u v \not \equiv u^{2}, v u \not \equiv u^{2}$. Já mostramos na página 18 que a dimensão de $I_{K^{*}}^{2}(G) / I_{K^{*}}^{3}(G)$ é 2 , portanto devemos ter que $u^{2}, v^{2} \in I_{K^{*}}^{3}(G)$, implicando que $\alpha_{1}^{2}+\alpha_{1} \alpha_{2}+\alpha_{2}^{2}=0$ e $\beta_{1}^{2}+\beta_{1} \beta_{2}+\beta_{2}^{2}=0$.

Note que $\alpha_{1}=0$ implica $\alpha_{2}=0$, donde $\Delta=0$ (contradição) (e análogo para $\left.\beta_{1}=0\right)$. Logo. $\alpha_{1} \neq 0$ e dividindo por $\alpha_{1}^{2}$ temos que $\left(\frac{\alpha_{2}}{\alpha_{1}}\right)^{2}+\left(\frac{\alpha_{2}}{\alpha_{1}}\right)+1=0$. Isto mostra que $\frac{\alpha_{2}}{\alpha_{1}}$ é raiz do polinômio $x^{2}+x+1$, i.e., é uma raiz cúbica primitiva da unidade. Conseqüentemente, para que a álgebra de grupo $K G$ tenha uma base multiplicativa filtrada é necessário que o corpo $K$ contenha uma raiz cúbica primitiva da unidade.

Mostraremos que esta condição também é suficiente construindo diretamente a base.

Seja $w \in K$ uma raiz cúbica primitiva da unidade. Escrevendo $\alpha_{2}=w \alpha_{1}$, de onde temos que $u=\alpha_{1}(1+a)+w \alpha_{2}(1+b)$ e da mesma forma deve $\operatorname{ser} \beta_{1}=w \beta_{2}$ ou $\beta_{1}=$ 
$w^{-1} \beta_{2}$. Como esta última implica $\Delta=0$ deve ser $\beta_{1}=w \beta_{2}$. Então, os elementos $u=\alpha_{1}[(1+a)+w(1+b)]$ e $v=\beta_{2}[w(1+a)+(1+b)]$ e fazendo $\alpha_{1}=\beta_{2}=1$ podemos escrever

$$
u=(1+a)+w(1+b) \quad \text { e } \quad v=w(1+a)+(1+b)
$$

e a seguir vamos determinar os outros elementos da base $B$.

Considerando o que foi demonstrado, a $K$-base multiplicativa filtrada $B$ já possui os seguintes elementos: 1, $u, v, u v, v u$, faltando apenas três elementos, que passaremos a determinar. Fixados $u=(a+1)+w(b+1)$ e $v=w(a+1)+(b+1)$ e reescrevendo as congruências (q), temos

$$
\begin{cases}u v \equiv w(a+1)(b+1)+w^{2}(a+1)^{2} & \left(\bmod I_{K^{-}}^{3}(G)\right) \\ v u \equiv w(a+1)(b+1)+(a+1)^{2} & \left(\bmod I_{K^{-}}^{3}(G)\right) \\ v^{2} \equiv 0 & \left(\bmod I_{K^{*}}^{3}(G)\right) \\ u^{2} \equiv 0 & \left(\bmod I_{K^{*}}^{3}(G)\right)\end{cases}
$$

Calculamos

$$
\begin{aligned}
u v u & \equiv w(a+1)^{3}+w^{2}(a+1)^{2}(b+1)\left(\bmod I_{K^{-}}^{4}(G)\right) \\
v u^{2} & \equiv 0 \quad\left(\bmod I_{K^{-}}^{4}(G)\right) \\
v u^{2} & \equiv 0 \quad\left(\bmod I_{K^{-}}^{4}(G)\right) \\
v u v & \equiv w^{2}(a+1)^{3}+w(a+1)^{2}(b+1)\left(\bmod I_{K^{-}}^{4}(G)\right) \\
v^{2} u & \equiv 0\left(\bmod I_{K^{-}}^{4}(G)\right) \\
v^{3} & \equiv 0\left(\bmod I_{K^{-}}^{4}(G)\right) \\
u^{3} & \equiv 0\left(\bmod I_{K^{-}}^{4}(G)\right) \\
u^{2} v & \equiv 0\left(\bmod I_{K^{*}}^{4}(G)\right)
\end{aligned}
$$


Como $\left|\begin{array}{cc}w & w^{2} \\ w^{2} & w\end{array}\right|=w^{2}+u \neq 0$, os elementos uvu e vuv são linearmente independentes $\bmod I_{K^{-}}^{4}(G)$. Calculamos agora

$$
\begin{aligned}
u v u^{2} & \equiv 0\left(\bmod I_{K^{5}}^{5}(G)\right) \\
u v u v & \equiv w^{2}(a+1)^{3}(b+1)\left(\bmod I_{K^{5}}^{5}(G)\right) \\
\text { vuvu } & \equiv w^{2}(a+1)^{3}(b+1)\left(\bmod I_{K^{*}}^{5}(G)\right) \\
\text { vuvv } & \equiv 0\left(\bmod I_{K^{5}}^{5}(G)\right),
\end{aligned}
$$

onde $u v u v \equiv v u v u\left(\bmod I_{K^{-}}^{5}(G)\right)$.

Considere o conjunto

$$
B=\{1, u, v, u v, v u, u v u, v u v, u v u v\},
$$

cuja matriz de coeficientes em relação à base ordenada $L$ é inversível

$$
\left(\begin{array}{cccccccc}
1 & 0 & 0 & 0 & 0 & 0 & 0 & 0 \\
0 & 1 & w & 0 & 0 & 0 & 0 & 0 \\
0 & w & 1 & 0 & 0 & 0 & 0 & 0 \\
0 & 0 & 0 & w^{2} & w & w^{2} & w^{2} & w^{2} \\
0 & 0 & 0 & 1 & w & w^{2} & w^{2} & w^{2} \\
0 & 0 & 0 & 0 & 0 & w & w^{2} & 1 \\
0 & 0 & 0 & 0 & 0 & w^{2} & w & 1 \\
0 & 0 & 0 & 0 & 0 & 0 & 0 & w^{2}
\end{array}\right)
$$

donde segue que $B$ é linearmente independente e, conseqüentemente, é uma base multiplicativa filtrada de $K G$.

Finalizando as construçòes apresentaremos para o grupo semi-diedral na observação seguinte que $I G$ possui uma base filtrada; no entanto, ela não é multiplicativa.

Observação: Neste caso é possivel definir uma $K$-base multiplicativa filtrada com $\tilde{u}=$ $u+\lambda(a+1)(b+1)$ e $\tilde{v}=v+\lambda(a+1)(b+1), \operatorname{com} \lambda \neq 0$ (vide referência [18]). 
Observação 3.2.3 Seja $G=\left\langle a, b \mid a^{2^{n}}=1, b^{2}=a^{2^{n-1}},{ }^{b} a=a^{-1+2^{n-1}}\right\rangle$, com $n>2$, um grupo de ordem $2^{n+1}$ e K um corpo de característica 2, então o conjunto

$$
B=\left\{(u v)^{i},(v u)^{i},(u v)^{i} u,(v u)^{i} v \mid i=0,1, \ldots, 2^{n-1}\right\}
$$

onde $u=a+b$ e $v=1+b$ é uma $K$-base filtrada de $K G$.

Demonstração: Inicialmente provamos a seguinte afirmação:

Afirmação: Nas condições acima vale:

(i) $(u v)^{i} \equiv(1+a)^{2 i-1}(1+b)\left(\bmod I_{K}^{2 i+1}(G)\right)$;

(ii) $(v u)^{i} \equiv(1+a)^{2 i-1}(1+b)+(1+a)^{2 i}\left(\bmod I_{K}^{2 i+1}(G)\right)$.

Demonstração da afirmação: Vamos provar os itens (i) e (ii) por indução em $i$.

(i) Para $i=1$ é imediato que $(u v)^{1}=[(1+a)+(1+b)](1+b)$. Note que $(1+b)^{2}=1+b^{2}=$ $1+a^{2^{n-1}}=(1+a)^{2^{n-1}} \in I_{K}^{3}(G)$. Portanto, $(u v)^{1} \equiv(1+a)(1+b)\left(\bmod I_{K^{k}}^{3}(G)\right)$. Por hipótese de indução, considere o item (i) verdadeiro para $i$ e vamos provar que vale para $i+1$, isto é, provamos que $(u v)^{i+1} \equiv(1+a)^{2 i+1}(1+b)\left(\bmod I_{K}^{2 i+3}(G)\right)$. De fato,

$$
(u v)^{i+1}=(u v)^{i}(u v) \equiv(1+a)^{2 i-1}(1+b)(1+a)(1+b)\left(\bmod I_{K}^{2 i+3}(G)\right) .
$$

Sabemos que

(A) $(1+b)(1+a) \equiv(1+a)(1+b)+(1+c)\left(\bmod I_{K^{\prime}}^{3}\left(G^{\prime}\right)\right)$ (calculado na p.39) 
e para o grupo dado temos que $c={ }^{b} a a^{-1}=a^{2^{n-1}-2}=a^{\left(2^{n-2}-1\right) 2}$, que implica

$$
\begin{aligned}
1+c & =\left(1+a^{2^{n-1}-1}\right)^{2}=\left((1+a)+(1+a)^{2}+\cdots+(1+a)^{2^{n-2}-1}\right)^{2} \\
& =(1+a)^{2}+(1+a)^{4}+\cdots+(1+a)^{2^{n-1}-2},
\end{aligned}
$$

donde temos que $(1+c) \equiv(1+a)^{2}\left(\bmod I_{K^{K}}^{3}(G)\right)$ e assim, substituindo em $(\mathrm{A})$ obtemos

$$
(1+b)(1+a) \equiv(1+a)(1+b)+(1+a)^{2}\left(\bmod I_{K}^{3}(G)\right) .
$$

Na expressão de $(u v)^{i+1}$, temos que

$$
\begin{aligned}
(u v)^{i+1} & \equiv(1+a)^{2 i-1}\left[(1+a)(1+b)+(1+a)^{2}\right](1+b)\left(\bmod I_{K}^{2 i+3}(G)\right) \\
& \equiv(1+a)^{2 i+1}(1+b)\left(\bmod I_{K^{K}}^{2 i+3}(G)\right),
\end{aligned}
$$

concluindo a prova da afirmação.

Da. mesma forma vamos provar (ii) por indução em $i$.

Para $i=1$, é imediato que

$$
\begin{aligned}
(v u)^{1} & \equiv(1+b)[(1+a)+(1+b)]\left(\bmod I_{K^{\prime}}^{3}(G)\right) \\
& \equiv(1+b)\left[(1+a)\left(\bmod I_{K^{\prime}}^{3}\left(G^{\prime}\right)\right)\right.
\end{aligned}
$$

e pelo já observado $(1+b)(1+a) \equiv(1+a)(1+b)+(1+a)^{2}\left(\bmod I_{K}^{3}(G)\right)$. Logo, na fórmula de $(v u)^{i}$, para $i=1$, temos que

$$
\begin{aligned}
(v u)^{1} & \equiv(1+a)^{2-1}(1+b)+(1+a)^{2-1}\left(\bmod I_{K}^{3}\left(G^{\prime}\right)\right) \\
& \equiv(1+a)(1+b)+(1+a)^{2}\left(\bmod I_{K}^{3}\left(G^{\prime}\right)\right)
\end{aligned}
$$

e a fórmula vale para $i=1$. 
Por hipótese de induçào. suponha que a fórmula vale para $i$ e vamos provar que vale para $i+1$, isto é, vale:

$$
(v u)^{i+1} \equiv(1+a)^{2 i+1}(1+b)+(1+a)^{2 i+2}\left(\bmod I_{K}^{2 i+3}(G)\right) .
$$

Com efeito,

$$
\begin{aligned}
(v u)^{i+1}= & (v u)^{i}(v u) \equiv\left[(1+a)^{2 i-1}(1+b)+(1+a)^{2 i}\right][(1+a)(1+b) \\
& \left.+(1+a)^{2}\right]\left(\bmod I_{K}^{2 i+3}(G)\right) \\
\equiv & (1+a)^{2 i-1}(1+b)(1+a)(1+b)+(1+a)^{2 i+1}(1+b) \\
& +(1+a)^{2 i-1}(1+b)(1+a)^{2}+(1+a)^{2 i+2}\left(\bmod I_{K}^{2 i+3}(G)\right) \\
\equiv & (1+a)^{2 i+1}(1+b)+(1+a)^{2 i+2}\left(\bmod I_{K}^{2 i+3}(G)\right),
\end{aligned}
$$

concluindo a prova do item (ii) da afirmação.

Agora, usando as fórmulas da afirmação escrevemos:

$$
\begin{aligned}
(u v)^{i} & \equiv(1+a)^{2 i-1}(1+b)\left(\bmod I_{K}^{2 i+1}(G)\right) \\
(v u)^{i} & \equiv(1+a)^{2 i-1}(1+b)+(1+a)^{2 i}\left(\bmod I_{K}^{2 i+1}(G)\right) \\
(u v)^{i} u & \equiv(1+a)^{2 i}(1+b)+(1+a)^{2 i+1}\left(\bmod I_{K}^{2 i+2}(G)\right) \\
(v u)^{i} v & \equiv(1+a)^{2 i}(1+b)\left(\bmod I_{K}^{2 i+2}(G)\right)
\end{aligned}
$$

onde $i=0,1, \ldots, 2^{n-1}$. Para todo $i$ os elementos $(v u)^{i}$ e $(v u)^{i}$ módulo $I_{K}^{2 i+1}(G)$ formam uma base sobre o corpo $K^{i}$ de $I_{K^{i}}^{2 i}(G) / I_{K}^{2 i+1}(G)$ e da mesma forma $(u v)^{i} u$ e $(v u)^{i} v$ módulo $I_{K}^{2 i+2}(G)$ forma uma base sobre o corpo $K^{\prime}$ de $I_{K}^{2 i+1}(G) / I_{K}^{2 i+2}(G)$.

Observamos que a matriz de coeficientes dos elementos do conjunto $B$ em relação à base ordenada $L$ é unimodular, pelo seguinte: 
Para $i=0$ temos que

$$
\left\{\begin{array}{l}
(u v)^{0}=(v u)^{0}=1=(1+a)^{0} \\
(u v)^{0} u=1 u=u=(1+a)^{1}+(1+b)^{1} \\
(v u)^{0} v=1 v=v=(1+b)^{1}
\end{array}\right.
$$

Para $i=2^{n-1}$ temos que

$$
(u v)^{2^{n-1}}=(v u)^{2^{n-1}} \equiv(1+a)^{2^{n}-1}(1+b)\left(\bmod I_{K}^{2^{n}+1}(G)\right)
$$

pois basta ver que

$$
(u v)^{2^{n-1}} \equiv(1+a)^{2^{n}-1}(1+b)\left(\bmod I_{K}^{2 i+1}(G)\right)
$$

e calculando

$$
\begin{aligned}
(v u)^{i} & \equiv(1+a)^{2 i-1}(1+b)+(1+a)^{2 i}\left(\bmod I_{K}^{2 i+1}(G)\right), \quad \text { para } i=2^{n-1}, \\
(v u)^{2^{n-1}} & \equiv(1+a)^{2^{n-1}}(1+b)+(1+a)^{2^{n}}\left(\bmod I_{K}^{2^{n}+1}(G)\right)
\end{aligned}
$$

e sendo $1+a^{2^{n}}=0$, temos

$$
(v u)^{2^{n-1}} \equiv(1+a)^{2^{n}-1}(1+b)\left(\bmod I_{K^{-}}^{2^{n}+1}(G)\right)
$$

e este é o último elemento da base $B$ pela ordenação de $L$.

Os outros elementos da base $B$ são os seguintes:

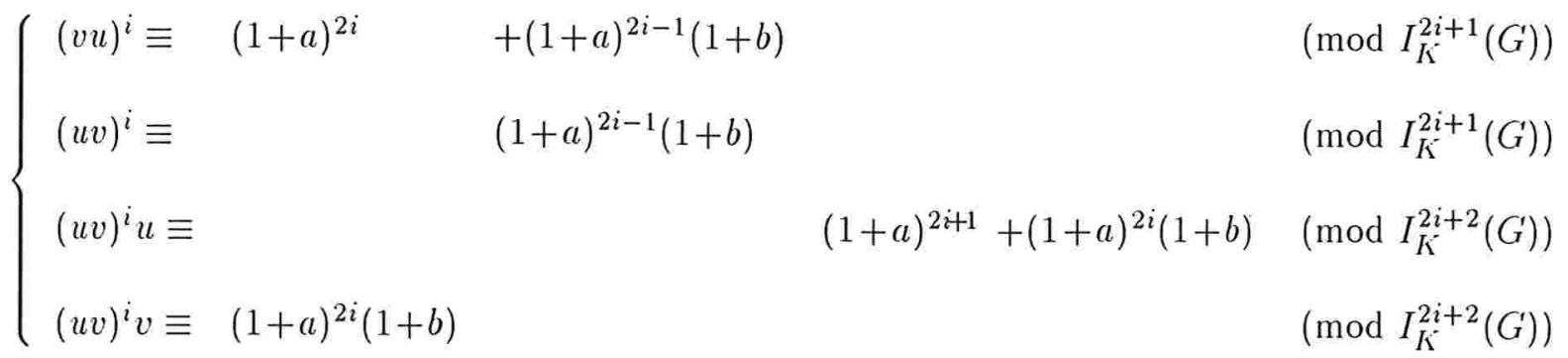

que forma a submatriz $A_{j}=\left(\begin{array}{llll}1 & 1 & * & * \\ 0 & 1 & * & * \\ 0 & 0 & 1 & 1 \\ 0 & 0 & 0 & 1\end{array}\right)$ que compõem, nesta ordem, a diagonal da matriz de coeficientes dos elementos do conjunto $B$ em relação à base ordenada $L$, onde $j=1, \ldots, 2^{n-1}-1$. A matriz é 


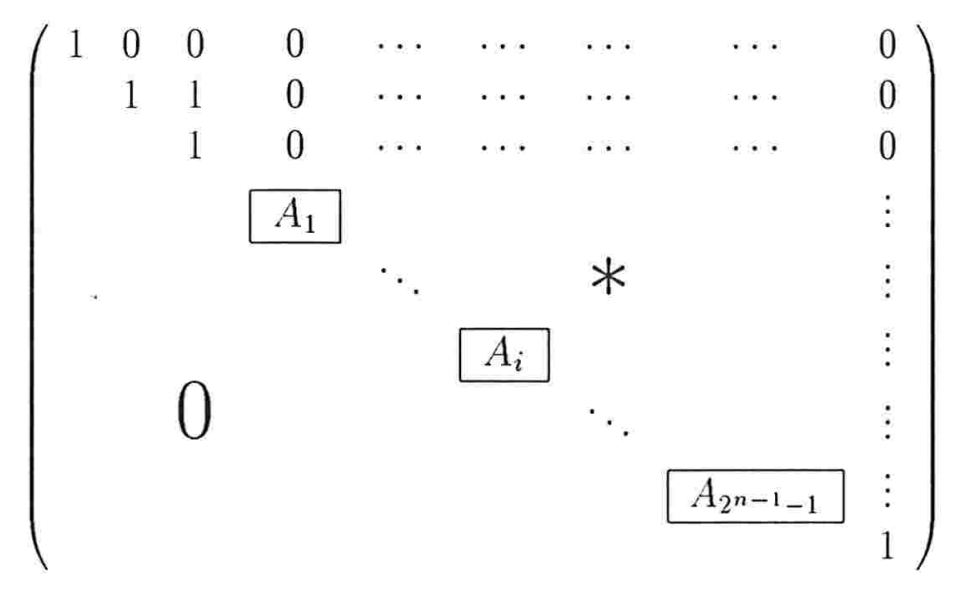

que é unimodular, portanto inversível e assim os elementos do conjunto

$$
B=\left\{(u v)^{i},(v u)^{i},(u v)^{i} u,(v u)^{i} v \mid i=0,1, \ldots, 2^{n-1}\right\}
$$

são linearmente independentes e de ordem igual à ordem de $G$. Portanto, conclui-se que $B$ é uma base de $K G$ sobre o corpo $K$. É imediato ver que $0 \neq v^{2}=(1+b)^{2}=(1+a)^{2^{n-1}} \notin B$, portanto $B \cup\{0\}$ não é fechado para multiplicação. 


\section{Capítulo 4}

\section{Exemplo de $p$-grupo não metacíclicos com base multiplicativa filtrada}

O objetivo deste capítulo é mostrar que as técnicas empregadas na demonstração do Teorema de Classificação no capítulo anterior podem ser aplicadas para determinação das bases multiplicativas filtradas de álgebras de grupo modulares para $p$-grupos nãometacíclicos. Apresentamos uma série de p-grupos $G$ não metacíclicos cujas álgebras de grupo $K G$ sobre um corpo $K$ de característica $p>0$ não admitem uma base multiplicativa filtrada, exceto num único caso onde $p=2$. Além deste exemplo, finalizamos este trabalho apresentando vários $p$-grupos finitos com três geradores cuja álgebra de grupo modular não possui uma tal base.

Inicialmente, provamos na Proposição 4.1.1 que os p-grupos introduzidos não são metacíclicos e estudamos o único caso onde $K^{K} G$ admite base multiplicativa filtrada. Concluímos este capítulo com mais alguns exemplos de $p$-grupos a três geradores cuja álgebra de grupo modular não possui uma base multiplicativa filtrada. 


\subsection{Exemplos especiais de álgebra de grupo com base multiplicativa filtrada}

Seja $p$ um primo e considere o $p$-grupo $G$ cuja apresentação é dada por

$$
G=\left\langle a, b \mid a^{p^{2 n}}=b^{p^{2 n}}=1,{ }^{b} a=b^{p^{n}} a^{p^{n}+1},{ }^{a} b=a^{p^{n}} b^{p^{n}+1},\left(a^{p^{n}}, b\right)=\left(b^{p^{n}}, a\right)=1\right\rangle
$$

$\operatorname{com} n \geq 1$.

Proposição 4.1.1 O grupo $G$ acima descrito tem as seguintes propriedades:

(i) Os elementos $a^{p^{n}}$ e $b^{p^{n}}$ estão no centro de $G$, isto é, $a^{p^{n}}, b^{p^{n}} \in Z(G)$;

(ii) O elemento central $a^{p^{n}} b^{p^{n}}$ é gerador do subgrupo comutador de $G$.

(iii) Para os elementos a e b de $G$ vale a fórmula:

$$
b^{k} a^{j}=a^{j k p^{n}+j} b^{j k p^{n}+k}, \quad \forall k, j \in \mathbb{N} .
$$

(iv) Todo elemento $g \in G$ pode ser escrito na forma $g=a^{i} b^{i}$, com $0 \leq i, j<p^{2 n}$. Além disso, a ordem de $G$ é $p^{4 n}$;

(v) G não é metacíclico.

\section{Demonstração:}


(i) Pela apresentação de $G$ os elementos $a^{p^{n}}$ e $b^{p^{n}}$ comutam com os geradores $a$ e $b$ do grupo $G$, logo comutam com todos os seus elementos.

(ii) Pela apresentação $G^{\prime}=\langle[b, a]\rangle$ e por um cálculo simples temos $c=[b, a]=b a b^{-1} a^{-1}=$ $b a a^{-1}=b^{p^{n}} a^{p^{n}+1} a^{-1}=b^{p^{n}} a^{p^{n}} \in Z(G)$.

(iii) Temos que $b a=c a b$ e por (ii), $c=a^{p^{n}} b^{p^{n}}, c \in Z\left(G^{\prime}\right)$, logo podemos escrever $b a=a c b=a a^{p^{n}} b^{p^{n}} b=a^{p^{n}} a b b^{p^{n}}$. Por indução sobre $k$ vemos que $b^{k} a=a^{k p^{n}+1} b^{k p^{n}+k}$ e fixando o valor de $k$, por indução sobre $j$ vamos provar que $b^{k} a^{j}=a^{j k p^{n}+j} b^{j k p^{n}+k}$. Para $j=1$, temos $b^{k} a=b^{k} a^{1}=a^{k p^{n}+1} b^{k p^{n}+k}$, como acima escrito. Por hipótese de indução o resultado vale para $j$ e para $j+1$ temos

$$
\begin{aligned}
b^{k} a^{j+1} & =b^{k} a^{j} a=a^{j k p^{n}+j} b^{j k p^{n}+k} a=a^{j k p^{n}+j} \\
& =a^{j k p^{n}+j} a^{\left(j k p^{n}+k\right) p^{n}+1} b^{\left(j k p^{n}+k\right) p^{n}+j k p^{n}+k} \\
& =a^{j k p^{n} j} a^{k p^{n}+1} b^{k p^{n}+j k p^{n}+k} \\
& =a^{(j+1) k p^{n}+(j+1)} b^{(j+1) k p^{n}+k} .
\end{aligned}
$$

(iv) Pelo que provamos no item anterior, qualquer elemento $g \in G$ dado por uma expressão finita com potência dos geradores $a$ e $b$ pode ser reescrita na forma $g=a^{i} b^{j}$, com $0 \leq i, j<p^{n}$. donde fica claro que $|G|=p^{4 n}$.

(v) Sabemos que todo $p$-grupo metacíclico não abeliano pode ser dado pela apresentação $H=\left\langle a, b \mid a^{p^{n}}=1, b^{p^{m}}=a^{p^{t}},{ }^{b} a=a^{r}\right\rangle$, onde vale $t \geq 0, r^{p^{m}} \equiv 1\left(\bmod p^{n}\right) \mathrm{e}$ $p^{t}(r-1) \equiv 0\left(\bmod p^{n}\right)$, onde o subgrupo comutador é $H^{\prime}=\langle[b, a]\rangle$ e $[b, a]=$ $b a b^{-1} a^{-1}={ }^{b} a a^{-1}=a^{r} a^{-1}$ e então $p$ divide $r-1$, pois caso contrário $H^{\prime}=\langle a\rangle$ implicaria $H / H^{\prime}$ cíclico e pelo resultado 2.1.3, $H$ seria abeliano (contradição). Isso permite concluir que $a^{r-1}=\left(a^{s}\right)^{p}$, com $a^{s} \in G$. Em vista deste resultado, basta ver que é impossivel termos que $a^{p^{n}} b^{p^{n}}=\left(a^{j} b^{k}\right)^{p}$ para algum $1 \leq j, k<p^{n} t^{\prime}$, o que 
provamos a seguir: Por aplicação da fórmula provada em (iii), podemos escrever

$$
a^{p^{n}} b^{p^{n}}=\left(a^{j} b^{k}\right)^{p}=\left(a^{j} b^{k}\right) \cdots\left(a^{j} b^{k}\right)=a^{j} \underbrace{\left(b^{k} a^{j}\right) \cdots\left(b^{k} a^{j}\right)}_{p-1 \text { vezes }} b^{k},
$$

donde vale a igualdade:

$$
a^{p^{n}} b^{p^{n}}=a^{\frac{p(p-1)}{2} j k p^{n}+p j} b^{\frac{p(p-1)}{2} j k p^{n}+p k},
$$

que implica

$$
a^{p^{n}-\frac{p(p-1)}{2} j k p^{n}-p j} b^{p^{n}-\frac{p(p-1)}{2} j k p^{n}-p k}=1 .
$$

Pelo item (iv), temos somente que

$$
\begin{cases}p^{n}-\frac{p(p-1)}{2} j k p^{n}-p j \equiv 0 & \left(\bmod p^{2 n}\right) \\ p^{n}-\frac{p(p-1)}{2} j k p^{n}-p k \equiv 0 & \left(\bmod p^{2 n}\right)\end{cases}
$$

Logo, multiplicando uma das equações do sistema por -1 e somando membro a membro obtemos que $j=k$, e substituindo numa das equações temos que a equação do segundo grau em $k\left(0 \leq k<p^{n}\right), \frac{p(p-1)}{2} p^{n} k^{2}+p k-p^{n}=0$ deve ter soluçòes inteiras e estas só podem ser da forma

$$
x^{\prime}= \pm p^{m_{1}} \quad \text { e } \quad x^{\prime \prime}= \pm p^{m_{2}}, \quad m_{1} ; m_{2} \geq 0 .
$$

Logo, $x^{\prime}+x^{\prime \prime}=-p$, isto é, $\pm\left(p^{m_{1}}+p^{m_{2}}\right)=-p$, donde temos $p^{m_{1}}+p^{m_{2}}=p$, que estudamos nos seguintes casos:

(1) $m_{1}=0$ e $m_{2}>0$, impossivel pois $1+p^{m_{2}}=p \Longrightarrow p \mid 1$;

(2) $m_{1}, m_{2}>0$, impossivel pois implicaria $2 \leq p^{m_{1}-1}+p^{m_{2}-1}=1$;

(3) Caso $m_{1}=m_{2}=0$. Na equação do segundo grau temos que $p^{n}=x^{\prime} \cdot x^{\prime \prime}=$ $p^{m_{1}} p^{m_{2}}=p^{0} p^{0}=1$, o que implica $n=0$ (impossivel, pois $n \geq 1$ ). 
Isto conclui a prova do item (v) e da proposição.

O lema que provaremos a seguir é uma versão do Lema 2.3.5 para álgebras de grupo $K G$ do grupo $G$ dado acima, para $p=2$ e $n=1$. Faremos uma demonstração construtiva.

Lema 4.1.2 Seja G o grupo dado pela apresentação

$$
G=\left\langle a, b \mid a^{4}=b^{4}=1, b a=b^{2} a^{3},{ }^{a} b=a^{2} b^{3},\left(a^{2}, b\right)=\left(b^{2}, a\right)=1\right\rangle
$$

e seja K um corpo de característica 2. Considere o conjunto

$$
L_{k}=\left\{(1+a)^{k_{1}}(1+b)^{k_{2}} \mid 0 \leq k_{1}<4 \quad \text { e } 0 \leq k_{2}<4, k_{1}+k_{2}=k\right\}
$$

e a projeção canônica $w: I_{k}(G) \rightarrow I_{K}^{k}(G) / I_{K}^{k+1}(G)$. Então a imagem de $L_{k}$ pela projeção canônica é uma base do quociente $I_{K}^{k}(G) / I_{K}^{k+1}(G)$ como espaço vetorial sobre $K$.

Demonstração: Faremos a demonstraçào em vários passos.

Afirmação 1: Seja $K G$ a álgebra de grupo acima considerada. Então vale a congruência

$$
(1+b)(1+a) \equiv(1+a)(1+b)+(1+a)^{2}+(1+b)^{2}\left(\bmod I_{K}^{3}(G)\right)
$$

onde os elementos $(1+a)^{2}$ e $(1+b)^{2}$ estào no centro de $K G$.

Demonstração: Seja $c=(b, a)$. Calculamos

$$
\begin{aligned}
(1+b)(1+a) & =(1+a)+b(1+a)=(1+a)+(b+b a)=(1+a)+(b+c a b) \\
& =(1+a)+(1+c a) b=(1+a)+(1+c a)(1+b)+(1+c a) .
\end{aligned}
$$


Aplicando a identidade (2.4.6) parra o termo $(1+c a)$, temos

$$
\begin{aligned}
(1+b)(1+a)= & (1+a)+((1+c)(1+a)+(1+c)+(1+a))(1+b) \\
& +((1+c)(1+a)+(1+c)+(1+a)) \\
= & (1+a)+(1+c)(1+a)(1+b)+(1+c)(1+b)+(1+a)(1+b) \\
& +(1+c)(1+c)+(1+c)+(1+a) \\
= & (1+c)(1+a)(1+b)+(1+c)(1+b)+(1+c)(1+b)+(1+a)(1+b) \\
& +(1+c)(1+c)+(1+c)
\end{aligned}
$$

Sabendo que $c \in G^{\prime} \leq \mathcal{D}_{2}\left(G^{\prime}\right)$ implica $1+c \in I_{K^{-}}^{2}\left(G_{r}^{\prime}\right)$, podemos escrever que

$$
(1+b)(1+a) \equiv(1+a)(1+b)+(1+c) \quad\left(\bmod I_{K^{K}}^{3}(G)\right)
$$

e para o grupo $G$ vale que $c=b a b^{-1} a^{-1}={ }^{b} a a^{-1}=\left(b^{2} a^{3}\right) a^{-1}=b^{2} a^{2}$. Substituindo na congruência obtemos que

$$
(1+b)(1+a) \equiv(1+a)(1+b)+\left(1+b^{2} a^{2}\right) \quad\left(\bmod I_{K^{-}}^{3}(G)\right)
$$

Aplicando a identidade (2.4.6) para o termo $\left(1+b^{2} a^{2}\right)$, temos

$$
(1+b)(1+a) \equiv(1+a)(1+b)+(1+b)^{2}(1+a)^{2}+(1+b)^{2}+(1+a)^{2} \quad\left(\bmod I_{K^{*}}^{3}(G)\right)
$$

Como $(1+a)^{2}(1+b)^{2} \in I_{K^{*}}^{3}(G)$, concluímos que

$$
(1+b)(1+a) \equiv(1+a)(1+b)+(1+b)^{2}+(1+a)^{2} \quad\left(\bmod I_{K}^{3}(G)\right)
$$

É claro que $(1+a)^{2}$ e $(1+b)^{2}$ são centrais em $K G$.

Afirmação 2: Seja $K G$ a álgebra de grupo acima considerada e seja $l$ um número inteiro não negativo. Então valem:

(i) se $l$ é par: $(1+b)(1+a)^{l}=(1+a)^{l}(1+b)$; 
(ii) se lé ímpar: $(1+b)(1+a)^{l} \equiv(1+a)^{l}(1+b)+(1+a)^{l-1}(1+b)^{2}+(1+a)^{l+1}\left(\bmod I_{K}^{l+2}(G)\right)$.

Demonstração: O item (i) segue trivialmente e para (ii) escrevemos $l=2 k+1, k \in \mathbb{N}$ e calculamos

$$
(1+b)(1+a)^{l}=(1+b)(1+a)^{2 k+1}=(1+b)(1+a)^{2 k}(1+a)
$$

e por (i)

$$
(1+b)(1+a)^{l}=(1+a)^{2 k}(1+b)(1+a),
$$

e pela Afirmação 1,

$$
\begin{aligned}
(1+b)(1+a)^{l} & \equiv(1+a)^{2 k}\left((1+a)(1+b)+(1+b)^{2}+(1+a)^{2}\right)\left(\bmod I_{K}^{l+2}(G)\right) \\
& \equiv(1+a)^{2 k+1}(1+b)+(1+a)^{2 k}(1+b)^{2}+(1+a)^{2 k+2}\left(\bmod I_{K}^{l+2}(G)\right),
\end{aligned}
$$

donde concluímos que

$$
(1+b)(1+a)^{l} \equiv(1+a)^{l}(1+b)+(1+a)^{l-1}(1+b)^{2}+(1+a)^{l+1}\left(\bmod I_{K}^{l+2}(G)\right) .
$$

Continuando a prova do Lema, procedemos como no Lema 2.4.5. Faremos indução sobre $k$, para provar que a imagem do conjunto $\left\{(1-a)^{l}(1-b)^{k-l} \mid 0 \leq l \leq k\right\}$ pela projeção canônica é um sistema de geradores do quociente $I_{K^{*}}^{k}\left(G^{\prime}\right) / I_{K^{*}}^{k+1}(G)$ como espaço vetorial sobre o corpo $K$. Seja $g, g^{\prime} \in G$, a identidade $1-g g^{\prime}=(1-g)+\left(1-g^{\prime}\right)-(1-g)\left(1-g^{\prime}\right)$ implica que a imagem do conjunto $\{(1-a),(1-b)\}$ pela projeçào canônica é um sistema de geradores do quociente $I_{K}(G) / I_{K}^{2}(G)$ como espaço vetorial sobre $K$. Suponhamos por hipótese de indução, que $w\left(L_{m}\right)$ com $m \leq k$ seja um sistema de geradores do espaço vetorial $I_{K^{-}}^{m}(G) / I_{K^{K}}^{m+1}(G)$ e vamos provar que as imagens dos produtos $u_{1} u_{2}$, com $u_{1} \in$ $I_{K}(G)$ e $u_{2} \in I_{K^{\prime}}^{m}(G)$ geram o quociente $I_{K^{\prime}}^{m+1}(G) / I_{K^{*}}^{m+2}(G)$ como espaço vetorial sobre $K$. 
Podemos escrever:

$$
\begin{aligned}
w\left(u_{1}\right) & \equiv \alpha_{1}(1-a)+\alpha_{2}(1-b)\left(\bmod I_{K^{-}}^{2}(G)\right), \operatorname{com} \alpha_{1}, \alpha_{2} \in K, \\
w\left(u_{2}\right) & \equiv \sum_{l=0}^{m} \beta_{l}(1-a)^{l}(1-b)^{m-l}\left(\bmod I_{K}^{m+1}(g)\right), \operatorname{com} \beta_{l} \in K, 0 \leq l \leq m .
\end{aligned}
$$

Portanto, temos o produto

$$
\begin{aligned}
(* *) \quad & w\left(u_{1} u_{2}\right) \equiv \sum_{l=0}^{m}\left(\alpha_{1} \beta_{l}(1+a)^{l+1}(1+b)^{m-l}\right. \\
& \left.+\alpha_{2} \beta_{l}(1+b)(1+a)^{l}(1+b)^{m-l}\right)\left(\bmod I_{K^{+}}^{m+2}(G)\right),
\end{aligned}
$$

onde substituindo-se os resultados para $l$ par e $l$ ímpar dados na afirmação acima obtemos:

$$
\begin{aligned}
w\left(u_{1} u_{2}\right) \equiv & \sum_{l=0}^{m} \alpha_{1} \beta_{l}(1+a)^{l+1}(1+b)^{m-l} \\
& +\sum_{l \text { par }}^{m} \alpha_{2} \beta_{l}(1+a)^{l}(1+b)^{m-l+1} \\
& +\sum_{l \text { impar }}^{m}\left(\alpha_{2} \beta_{l}\left[(1+a)^{l}(1+b)+(1+a)^{l-1}(1+b)^{2}+(1+a)^{l+1}\right](1+b)^{m-l}\right) \\
& \left(\bmod I_{K^{m}}^{m+2}\left(G^{\prime}\right)\right)
\end{aligned}
$$

ou melhor

$$
\begin{aligned}
w\left(u_{1} u_{2}\right) \equiv & \sum_{l=0}^{m} \alpha_{1} \beta_{l}(1+a)^{l+1}(1+b)^{m-l} \\
& +\sum_{l \text { par }}^{m} \alpha_{2} 3_{l}(1+a)^{l}(1+b)^{m-l+1} \\
& +\sum_{l \text { impar }}^{m} \alpha_{2} \beta_{l}\left((1+a)^{l}(1+b)^{m-l+1}+(1+a)^{l-1}(1+b)^{m-l+2}\right. \\
& \left.+(1+a)^{l+1}(1+b)^{m-l}\right) \quad\left(\bmod I_{K^{m}}^{m+2}(G)\right)
\end{aligned}
$$

Observemos os elementos $w\left(u_{1} u_{2}\right)$ são combinação linear dos termos da forma

$$
(1+a)^{k_{1}}(1+b)^{k_{2}}
$$


com $k_{1}+k_{2}=m+1$. Concluímos que as imagens dos produtos $(1+a)^{k_{1}}(1+b)^{k_{2}}$ pela projeção canônica geram o quociente $I_{K}^{m+1}(G) / I_{K}^{m+2}(G)$ como espaço vetorial sobre $K$. Já provamos na página 22 que $w\left(L_{K^{*}}\right)$ é linearmente independente sobre $K$. Podemos escrever

$$
L=\left\{(1+a)^{k_{1}}(1+b)^{k_{2}} \mid 0 \leq k_{1}<p^{2 n} \quad \text { e } \quad 0 \leq k_{2}<p^{2 n}\right\}=\bigcup_{k=0}^{2 p^{2 n}-2} L_{k}
$$

e claramente $L$ é uma base da álgebra $K G$ sobre $K$, pois $|G|=|L|$. E a mesma orientação anterior pode ser dada à base $L$.

Aplicamos o Lema para o grupo $G$ dado e facilmente obtemos o seguinte quadro:

\begin{tabular}{|c|l|c|}
\hline$K$-dimensão & \multicolumn{1}{|c|}{$K$-base } & Quocientes \\
\hline 1 & 1 & $I^{0} / I^{1}$ \\
\hline 2 & $(1+a) ;(1+b)$ & $I / I^{2}$ \\
\hline 3 & $(1+a)^{2} ;(1+a)(1+b) ;(1+b)^{2}$ & $I^{2} / I^{3}$ \\
\hline 4 & $(1+a)^{3} ;(1+a)^{2}(1+b) ;(1+a)(1+b)^{2} ;(1+b)^{3}$ & $I^{3} / I^{4}$ \\
\hline 3 & $(1+a)^{3}(1+b) ;(1+a)^{2}(1+b)^{2} ;(1+a)(1+b)^{3}$ & $I^{4} / I^{5}$ \\
\hline 2 & $(1+a)^{3}(1+b)^{2} ;(1+a)^{2}(1+b)^{3}$ & $I^{5} / I^{6}$ \\
\hline 1 & $(1+a)^{3}(1+b)^{3}$ & $I^{6} / I^{7}$ \\
\hline
\end{tabular}

Proposição 4.1.3 A álgebra de grupo modular $K G$ sobre um corpo $K$ de característica 2 tem uma Kr-base filtrada dada pelo conjunto

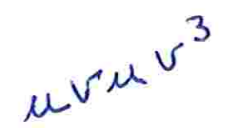

$$
\begin{gathered}
B=\left\{1, u, v, u v, v u, v^{2}, u v u, u v^{2}, v u v, v^{3}, u v u v, u v^{3}, v u v_{d}^{2}, u v u v^{2}, v u v^{3} \mid u=a+b,\right. \\
\left.v=\mu_{1} a+\mu_{2} b+\left(\mu_{1}+\mu_{2}\right) \quad \text { e } \quad \mu_{1}, \mu_{2} \in K \quad \text { e } \quad \mu_{1} \neq \mu_{2}\right\} .
\end{gathered}
$$

Particularmente, $B$ é uma $K$-base multiplicativa filtrada fazendo $\mu_{1}=1$ e $\mu_{2}=0$, onde $v=1+a$ 
Demonstração: (De forma análoga ao que fizemos no final da página 34 para o Teorema 3.1.1.) Sejam $u, v$ elementos de $K G$ tais que

$$
\begin{aligned}
& u=\alpha_{1}(a+1)+\alpha_{2}(b+1)\left(\bmod I_{K^{\prime}}^{2}\left(G^{\prime}\right)\right) \\
& v=\beta_{1}(a+1)+\beta_{2}(b+1)\left(\bmod I_{K^{-}}^{2}(G)\right),
\end{aligned}
$$

com $\Delta=a_{1} \beta_{2}+\beta_{1} \alpha_{2} \neq 0$. Por multiplicação, obtemos:

$u v \equiv\left[\alpha_{1}(a+1)+\alpha_{2}(b+1)\right]\left[\beta_{1}(a+1)+\beta_{2}(b+1)\right]\left(\bmod I_{K^{\prime}}^{3}(G)\right)$

$u v \equiv \beta_{1} \alpha_{1}(a+1)^{2}+\beta_{1} \alpha_{2}(b+1)(a+1)+\beta_{2} \alpha_{1}(a+1)(b+1)+\beta_{2} \alpha_{2}(b+1)^{2}\left(\bmod I_{K^{*}}^{3}(G)\right)$.

Usando a fórmula (4.1), podemos calcular $\bmod I_{K^{(}}^{3}(G)$ e obter as seguintes congruências, $\bmod I_{K}^{3}(G):$

$$
\begin{aligned}
u v & \equiv\left(\alpha_{1}+\alpha_{2}\right) \beta_{1}(1+a)^{2}+\Delta(1+a)(1+b)+\alpha_{2}\left(\beta_{1}+\beta_{2}\right)(1+b)^{2} \\
v u & \equiv \alpha_{1}\left(\beta_{1}+\beta_{2}\right)(1+a)^{2}+\Delta(1+a)(1+b)+\beta_{2}\left(\alpha_{1}+\alpha_{2}\right)(1+b)^{2} \\
u^{2} & \equiv\left(\alpha_{1}+\alpha_{2}\right) \alpha_{1}(1+a)^{2}+\alpha_{2}\left(\alpha_{1}+\alpha_{2}\right)(1+b)^{2}: \\
v^{2} & \equiv\left(\beta_{1}+\beta_{2}\right) \beta_{1}(1+a)^{2}+\beta_{2}\left(\beta_{1}+\beta_{2}\right)(1+b)^{2} .
\end{aligned}
$$

Na tabela da página anterior, sabemos que a $K^{\circ}$-dimensão do quociente $I_{K^{j}}^{2}(G) / I_{K^{j}}^{3}(G)$ é igual a 3 e pelas congruências $(4.1)-(4.4)$, observa-se que $u v \not \equiv v u\left(\bmod I_{K}^{3}(G)\right), u v \not \equiv$ $u^{2}\left(\bmod I_{K^{-}}^{3}(G)\right), u v \not \equiv v^{2}\left(\bmod I_{K^{-}}^{3}(G)\right), v u \not \equiv u^{2}\left(\bmod I_{K^{\prime}}^{3}(G)\right)$ e $v u \not \equiv v^{2}\left(\bmod I_{K^{*}}^{3}(G)\right)$. Para que este conjunto seja uma base, necessariamente deve acontecer um dos seguintes casos:

Caso 1: $u^{2} \equiv v^{2}\left(\bmod I_{K^{(}}^{3}(G)\right)$ e $v^{2}, u^{2} \notin I_{K^{-}}^{3}(G)$, que pelas congruências faz $\alpha_{2}+\alpha_{1} \alpha_{2}=$ $3_{1}^{2}+\beta_{1} \beta_{2}$ e $\alpha_{2}^{2}+\alpha_{1} \alpha_{2}=\beta_{2}^{2}+\beta_{1} \beta_{2}$. Daí temos $\left(\alpha_{1}+\alpha_{2}\right)^{2}=\left(\beta_{1}+\beta_{2}\right)^{2}$ e então $\alpha_{1}+\alpha_{2}=\beta_{1}+\beta_{2}$. Escrevemos a segunda igualdade na forma $\alpha_{2}\left(\alpha_{1}+\alpha_{2}\right)=\beta_{2}\left(\beta_{1}+\beta_{2}\right) \mathrm{e}$ 
substituindo temos $\alpha_{2}\left(\beta_{1}+\beta_{2}\right)=\beta_{2}\left(\alpha_{1}+\alpha_{2}\right)$, que implica em $\alpha_{2} \beta_{1}+\alpha_{2} \beta_{2}=\beta_{2} \alpha_{1}+\beta_{2} \alpha_{2}$. Portanto, $\Delta=\alpha_{2} \beta_{1}+\beta_{2} \alpha_{1}=0$, contradição! Logo, $u^{2} \equiv v^{2}\left(\bmod I_{K^{-}}^{3}(G)\right)$ é impossível.

Caso 2: $u^{2} \equiv 0\left(\bmod I_{K^{3}}^{3}(G)\right.$ ou $v^{2} \equiv 0\left(\bmod I_{K^{*}}(G)\right)$

Se $u^{2} \equiv 0\left(\bmod I_{K^{K}}^{3}(G)\right)$ temos que $\left(\alpha_{1}+\alpha_{2}\right) \alpha_{1}=0$ e $\alpha_{2}\left(\alpha_{1}+\alpha_{2}\right)=0$, pois $\left\{(1+a)^{2} \bmod I_{K^{(}}^{3}(G),(1+b)^{2} \bmod I_{K^{*}}^{3}(G)\right\}$ são linearmente independentes no quociente $I_{K^{\prime}}^{2}(G) / I_{K^{\prime}}^{3}(G)$ como espaço vetorial sobre $K$. Conseqüentemente, se $\alpha_{1} \neq \alpha_{2}$ teríamos que $\alpha_{1}=\alpha_{2}=0$, contradição. Logo, só resta o caso em que $\alpha_{1}=\alpha_{2} \neq 0$. Veremos que neste caso se obtém uma base de $I_{K^{*}}^{2}(G) / I_{K}^{3}(G)$. Pelas congruências $(4.1)$ - (4.4), nesta situação obtemos

$$
\begin{cases}u v \equiv \lambda(1+a)(1+b)+\lambda(1+b)^{2} & \left(\bmod I_{K}^{3}(G)\right) \\ v u \equiv \lambda(1+a)^{2}+\lambda(1+a)(1+b) & \left(\bmod I_{K^{\prime}}^{3}(G)\right) \\ v^{2} \equiv \lambda \beta_{1}(1+a)^{2}+\lambda \beta_{2}(1+b)^{2} & \left(\bmod I_{K^{3}}^{3}(G)\right)\end{cases}
$$

$\operatorname{com} \lambda=\beta_{1}+\beta_{2} \neq 0$. A matriz de coeficientes destes elementos na base

$$
w\left(\left\{(1+a)^{2},(1+a)(1+b),(1+b)^{2}\right\}\right)
$$

é $\left(\begin{array}{ccc}0 & \lambda & \lambda \\ \lambda & \lambda & 0 \\ \lambda \beta_{1} & 0 & \lambda \beta_{2}\end{array}\right)$ que tem determinante igual a $\lambda^{4} \neq 0$. Portanto, $u v, v u$ e $v^{2}$ formam uma base de $I_{K^{j}}^{2}\left(G^{\prime}\right) / I_{K^{\prime}}^{3}(G)$ como espaço vetorial sobre $K$.

Multiplicando estes elementos por $u$ e $v$ tomando os resultados módulo $I_{K^{-}}^{4}(G)$, obtemos:

$$
\left\{\begin{array}{l}
u v u \equiv \lambda\left[(1+a)^{3}+(1+a)^{2}(1+b)+(1+a)(1+b)^{2}+(1+b)^{3}\right] \\
u v^{2} \equiv \lambda\left[\beta_{1}(1+a)^{3}+\beta_{1}(1+a)^{2}(1+b)+\beta_{2}(1+a)(1+b)^{2}+\beta_{2}(1+b)^{3}\right] \\
v u v \equiv \lambda^{2}\left[(1+a)^{2}(1+b)+(1+a)(1+b)^{2}\right] \\
v^{3} \equiv \lambda\left[\beta_{1}^{2}(1+a)^{3}+\beta_{1} \beta_{2}(1+a)^{2}(1+b)+\beta_{1} \beta_{2}(1+a)(1+b)^{2}+\beta_{2}^{2}(1+b)^{3}\right]
\end{array}\right.
$$


Vemos que formam uma base de $I_{K^{*}}^{3}(G) / I_{K^{*}}^{4}(G)$ como espaço vetorial sobre $K$. Com efeito, eles são linearmente independentes, pois a matriz dos coeficientes em relação à base dada pela imagem da projeção canônica de $L_{3}=\left\{(1+a)^{3},(1+a)^{2}(1+b),(1+a)(1+b)^{2},(1+b)^{3}\right\}$ é dada por

$$
\left(\begin{array}{cccc}
\lambda & \lambda & \lambda & \lambda \\
\lambda \beta_{1} & \lambda \beta_{1} & \lambda \beta_{2} & \lambda \beta_{2} \\
0 & \lambda^{2} & \lambda^{2} & 0 \\
\lambda \beta_{1}^{2} & \lambda \beta_{1} \beta_{2} & \lambda \beta_{1} \beta_{2} & \lambda \beta_{2}^{2}
\end{array}\right)
$$

que tem determinante igual a $\lambda^{8} \neq 0$. Como seu número é igual à dimensão, eles formam uma base.

Multiplicando agora estes elementos por $u$ e $v$ e tomando os resultados módulo $I_{K}^{5}(G)$, obtemos

$$
\left\{\begin{array}{l}
u v u v \equiv \lambda^{2}\left[(1+a)^{3}(1+b)+(1+a)^{2}(1+b)^{2}+(1+a)(1+b)^{3}\right] \\
u v^{3} \equiv \lambda^{2}\left[\beta_{1}(1+a)^{3}(1+b)+\beta_{1}(1+a)^{2}(1+b)^{2}+\beta_{2}(1+a)(1+b)^{3}\right] \\
v u v^{2} \equiv \lambda^{2}\left[\beta_{1}(1+a)^{3}(1+b)+\beta_{2}(1+a)^{2}(1+b)^{2}+\beta_{2}(1+a)(1+b)^{3}\right]
\end{array}\right.
$$

Da mesma forma, a matriz dos coeficientes é

$$
\left(\begin{array}{ccc}
\lambda^{2} & \lambda^{2} & \lambda^{2} \\
\lambda^{2} \beta_{1} & \lambda^{2} \beta_{1} & \lambda^{2} \beta_{1} \\
\lambda^{2} \beta_{1} & \lambda^{2} \beta_{2} & \lambda^{2} \beta_{2}
\end{array}\right)
$$

que tem determinante $\lambda^{8} \neq 0$. e novamente os elementos acima formam uma base do quociente $I_{K^{*}}^{4}(G) / I_{K^{j}}^{5}(G)$ como espaço vetorial sobre $K^{*}$.

Do mesmo modo, multiplicando por $u$ e $v$ e tomando módulo $I_{K^{-}}^{6}(G)$, obtemos

$$
\left\{\begin{array}{l}
u v u v^{2} \equiv \lambda^{2}\left[(1+a)^{3}(1+b)^{2}+(1+a)^{2}(1+b)^{3}\right] \\
v u v^{3} \equiv \lambda^{2}\left[3_{1}(1+a)^{3}(1+b)^{2}+\beta_{2}(1+a)^{2}(1+b)^{3}\right]
\end{array}\right.
$$


Da mesma forma a matriz dos coeficientes $\left(\begin{array}{cc}\lambda^{2} & \lambda^{2} \\ \lambda^{2} \beta_{1} & \lambda^{2} \beta_{2}\end{array}\right)$ tem determinante $\lambda^{5} \neq 0$, os elementos acima formam uma base do espaço $I_{K^{(}}^{5}(G) / I_{K^{(}}^{6}(G)$.

E, finalmente, do mesmo modo obtemos que

$$
u v u v^{3} \equiv \lambda^{3}(1+a)^{3}(1+b)^{3}\left(\bmod I_{K}^{\tau}(G)\right) .
$$

E desde que o número de elementos linearmente independents tomados módulo $I_{K}^{j}(G)$, $\operatorname{com} j=0,1,2, \ldots, 7$, iguais aos números das $K$-dimensões dos quocientes $I_{K^{*}}^{j}(G) / I_{K^{\prime}}^{j+1}(G)$, podemos concluir que os elementos

$$
\left\{1, u, v, u v, v u, v^{2}, u v u, u v^{2}, v u v, v^{3}, u v u v, u v^{3}, v u v^{2}, u v u v^{2}, v u v^{2}, u v u v^{3}\right\}
$$

formam uma $K$-base filtrada para a álgebra de grupo $K G$.

Calculando temos que $u v u=\left(\mu_{1}+\mu_{2}\right) u^{2}, \operatorname{logo} 0 \neq u^{2} \in B$ se $\mu_{1}+\mu_{2}=1$ e a escolha $\mu_{1}=1$ e $\mu_{2}=0$ faz $B \cup\{0\}$ um semi-grupo para multiplicação de $K G$ (vide Anexo).

Com respeito aos p-grupos $G$ dados pela apresentação acima, concluímos esta seção provando o seguinte teorema:

Teorema 4.1.4 Seja G o p-grupo não metacíclico dado acima e considere $K$ um corpo de característica $p>0$. Então a álgebra de grupo modular $K G$ não possui uma base multiplicativa filtrada sobre $K$, exceto no único caso em que $p=2$ e $n=1$.

Demonstração: Calculamos $c=b a b^{-1} a^{-1}={ }^{b} a a^{-1}=b^{p^{n}} a^{p^{n}+1} a^{-1}=b^{p^{n}} a^{p^{n}} \in Z(G)$. Logo temos $1-c=1-b^{p^{n}} a^{p^{n}}$ e que pela identidade (2.4.6) escrevemos

$$
1-c=(1-b)^{p^{n}}+(1-a)^{p^{n}}-(1-b)^{p^{n}}(1-a)^{p^{n}}
$$


Se $p>2$ e $n>1$, segue que $1-c \in I_{K}^{3}(G)$. Na demonstração do Teorema Principal na página 36 observamos que este fato contradiz a existência de bases multiplicativas filtradas. O único caso possível é $p=2$ e $n=1$. que foi estudado na proposição acima.

Finalizamos este capítulo, apresentando uma classe de $p$-grupos com três geradores não abelianos que não possuem base multiplicativa filtrada.

Teorema 4.1.5 Seja G um p-grupo finito cuja apresentação é dada por

$$
G=\left\langle x, y, z \mid(x, z)=(y, z)=1,(x, y)=z^{p^{n}}\right\rangle
$$

e seja $K$ um corpo de característica $p>0$. Então a álgebra de grupo $K G$ não admite uma base multiplicativa filtrada se $p^{n} \geq 3$.

Demonstração: Seja $1 \cup B$ uma base multiplicativa filtrada de $K G$, então $B$ é uma base filtrada do radical $I_{K^{*}}(G)$. Um argumento análogo ao dado no Lema 2.4.5, mostra que as imagens de $1-x, 1-y, 1-z$ pela projeção canônica formam uma base de $I_{K^{*}}(G) / I_{K^{*}}^{2}\left(G^{\prime}\right)$ sobre o corpo $K$. Pelo resultado $(2.2 .5)$ a subálgebra de Frattini de $I_{K}(G)$ é $I_{K^{K}}^{2}(G)$. Logo os elementos de $B \backslash I_{K^{(}}^{2}(G)$ são geradores de $I_{K^{-}}(G)$ sobre $K$ e denotando estes por $u, v, w$, podemos escrever $K[u, v, w]=I_{K}(G)$ que é não comutativa, pois $G$ é um grupo não comutativo.

\section{Escrevemos}

$$
\begin{cases}u=\alpha_{1}(1-x)+\alpha_{2}(1-y)+\alpha_{3}(1-z) & \left(\bmod I_{K}^{2}(G)\right. \\ v=\beta_{1}(1-x)+\beta_{2}(1-y)+\beta_{3}(1-z) & \left(\bmod I_{K}^{2}(G)\right. \\ w=\gamma_{1}(1-x)+\gamma_{2}(1-y)+\gamma_{3}(1-z) & \left(\bmod I_{K}^{2}(G)\right.\end{cases}
$$




$$
\begin{aligned}
\operatorname{com} \Delta= & \left|\begin{array}{ccc}
\alpha_{1} & \alpha_{2} & \alpha_{3} \\
\beta_{1} & \beta_{2} & \beta_{3} \\
\gamma_{1} & \gamma_{2} & \gamma_{3}
\end{array}\right| \neq 0 \text {, donde calculamos } \\
u v \equiv & \beta_{1} \alpha_{1}(1-x)^{2}+\beta_{1} \alpha_{2}(1-y)(1-x)+\beta_{1} \alpha_{3}(1-z)(1-x) \\
& +\beta_{2} \alpha_{1}(1-x)(1-y)+\beta_{2} \alpha_{2}(1-y)^{2}+\beta_{2} \alpha_{3}(1-z)(1-y) \\
& +\beta_{3} \alpha_{1}(1-x)(1-z)+\beta_{3} \alpha_{2}(1-y)(1-z)+\beta_{3} \alpha_{3}(1-z)^{2} \quad\left(\bmod I_{K}^{3}(G)\right) .
\end{aligned}
$$

Pela apresentação do grupo temos que $c=[x, y]=z^{p^{n}}$ e que valem as seguintes relações:

$$
\begin{aligned}
& (1-z)(1-x)=(1-x)(1-z), \\
& (1-z)(1-y)=(1-y)(1-z),
\end{aligned}
$$

pois $z \in Z(G) \mathrm{e}$

$$
\begin{aligned}
(1-y)(1-x) & =(1-x)-y(1-x)=(1-x)-(y-y x)=(1-x)-(y-c x y) \\
& =(1-x)-(1-c x) y=(1-x)+(1-c x)(1-y)-(1-c x) .
\end{aligned}
$$

Pela identidade (2.4.6) e por $(1-c) \in I_{K^{-}}^{2}(G)$, temos que

$$
\begin{aligned}
(1-y)(1-x)= & (1-x)+((1-c)+(1-x)-(1-c)(1-x))(1-y) \\
& -((1-c)+(1-x)-(1-c)(1-x)) \\
= & (1-c)(1-y)+(1-x)(1-y)-(1-c)(1-x)(1-y) \\
& -(1-c)-(1-x)+(1-c)(1-x) \\
= & (1-x)(1-y)-(1-c)\left(\bmod I_{K}^{3}(G)\right) .
\end{aligned}
$$

Substituindo $c=z^{p^{n}}, p^{n} \geq 3$, temos

$$
1-c=1-z^{p^{k}}=(1-z)^{p^{k}} \in I_{K^{*}}^{3}(G),
$$


o que implica

$$
(1-y)(1-x) \equiv(1-x)(1-y) \quad\left(\bmod I_{K^{(}}^{3}(G)\right) .
$$

Continuando o cálculo do produto $u v$, obtemos, $\left(\bmod I_{K}^{3}(G)\right)$,

$$
\left\{\begin{array}{l}
u v \equiv \beta_{1} \alpha_{1}(1-x)^{2}+\beta_{2} \alpha_{2}(1-y)^{2}+\beta_{3} \alpha_{3}(1-z)^{2}+\left(\beta_{1} \alpha_{2}+\alpha_{1} \beta_{2}\right)(1-x)(1-y) \\
+\left(\beta_{1} \alpha_{3}+\alpha_{1} \beta_{3}\right)(1-x)(1-z)+\left(\beta_{2} \alpha_{3}+\alpha_{2} \beta_{3}\right)(1-y)(1-z) \\
v u \equiv \alpha_{1} \beta_{1}(1-x)^{2}+\alpha_{2} \beta_{2}(1-y)^{2}+\alpha_{3} \beta_{3}(1-z)^{2}+\left(\beta_{1} \alpha_{2}+\alpha_{1} \beta_{2}\right)(1-x)(1-y) \\
+\left(\beta_{1} \alpha_{3}+\alpha_{1} \beta_{3}\right)(1-x)(1-z)+\left(\beta_{2} \alpha_{3}+\alpha_{2} \beta_{3}\right)(1-y)(1-z)
\end{array}\right.
$$

Analogamente,

$$
\left\{\begin{array}{l}
u w \equiv \gamma_{1} \alpha_{1}(1-x)^{2}+\gamma_{2} \alpha_{2}(1-y)^{2}+\gamma_{3} \alpha_{3}(1-z)^{2}+\left(\gamma_{1} \alpha_{2}+\alpha_{2} \gamma_{2}\right)(1-x)(1-y) \\
\left(\gamma_{1} \alpha_{3}+\alpha_{1} \gamma_{3}\right)(1-x)(1-z)+\left(\gamma_{2} \alpha_{3}+\alpha_{2} \gamma_{3}\right)(1-y)(1-z) \\
w u \equiv \alpha_{1} \gamma_{1}(1-x)^{2}+\alpha_{2} \gamma_{2}(1-y)^{2}+\alpha_{3} \gamma_{3}(1-z)^{2}+\left(\gamma_{1} \alpha_{2}+\alpha_{2} \gamma_{1}\right)(1-x)(1-y) \\
\left(\gamma_{1} \alpha_{3}+\alpha_{1} \gamma_{3}\right)(1-x)(1-z)+\left(\gamma_{2} \alpha_{3}+\alpha_{2} \gamma_{3}\right)(1-y)(1-z)
\end{array}\right.
$$

e

$$
\left\{\begin{array}{l}
v w \equiv \gamma_{1} \beta_{1}(1-x)^{2}+\gamma_{2} \beta_{2}(1-y)^{2}+\gamma_{3} \beta_{3}(1-z)^{2}+\left(\gamma_{1} \beta_{2}+\beta_{1} \gamma_{2}\right)(1-x)(1-y) \\
\left(\gamma_{1} \beta_{3}+\beta_{1} \gamma_{3}\right)(1-x)(1-z)+\left(\gamma_{2} \beta_{3}+\beta_{2} \gamma_{3}\right)(1-y)(1-z) \\
w v \equiv \beta_{1} \gamma_{1}(1-x)^{2}+\beta_{2} \gamma_{2}(1-y)^{2}+\beta_{3} \gamma_{3}(1-z)^{2}+\left(\gamma_{1} \beta_{2}+\beta_{1} \gamma_{2}\right)(1-x)(1-y) \\
\left(\gamma_{1} \beta_{3}+\beta_{1} \gamma_{3}\right)(1-x)(1-z)+\left(\gamma_{2} \beta_{3}+\beta_{2} \gamma_{3}\right)(1-y)(1-z)
\end{array}\right.
$$

Segue diretamente que

$$
\begin{cases}u v \equiv v u & \left(\bmod I_{K^{(}}^{3}(G)\right) \\ u w \equiv v u & \left(\bmod I_{K^{(}}^{3}(G)\right) \\ v w \equiv v w & \left(\bmod I_{K^{(}}^{3}(G)\right) .\end{cases}
$$

Se $p=2$ temos que

$$
u v, v u, u w, w u, v w, w v \notin I_{K^{(}}^{3}(G),
$$

pois caso contrário

$$
\left|\begin{array}{ll}
\alpha_{1} & \alpha_{2} \\
\beta_{1} & \beta_{2}
\end{array}\right|=\left|\begin{array}{cc}
\alpha_{1} & \alpha_{3} \\
\beta_{1} & \beta_{3}
\end{array}\right|=\left|\begin{array}{cc}
\alpha_{2} & \alpha_{3} \\
\beta_{2} & \beta_{3}
\end{array}\right|=0
$$

e pela conhecida regra de Laplace, $\Delta=0$, contradição! 
Se $p>2$, também temos que

$$
u v, v u, u w, w u, v w, w v \notin I_{K}^{3}(G),
$$

pois caso contrário $u v \equiv 0\left(\bmod I_{K}^{3}(G)\right)$ e como $(1-x)^{2},(1-y)^{2},(1-z)^{2},(1-x)(1-y)$, $(1-x)(1-z),(1-y)(1-z)$ todos $\bmod I_{K^{(}}^{3}(G)$ formam uma base do quociente $I_{K^{*}}^{2}(G) / I_{K^{\prime}}^{3}(G)$ como espaço vetorial sobre $K$, temos as igualdades: $\beta_{1} \alpha_{1}=0, \beta_{2} \alpha_{2}=0, \beta_{3} \alpha_{3}=0$, $3_{1} \alpha_{2}+\alpha_{1} \beta_{2}=0, \beta_{1} \alpha_{3}+\alpha_{1} \beta_{3}=0$ e $\beta_{2} \alpha_{3}+\beta_{3} \alpha_{2}=0$. Claramente não podemos ter $3_{1}=\beta_{2}=\beta_{3}=0$. Suponhamos então, sem perda de generalidade, que $\beta_{1} \neq 0$, o que implica na primeira equação $\alpha_{1}=0$ e substituindo nas outras igualdades, temos

$$
\left\{\begin{array} { l } 
{ \beta _ { 1 } \alpha _ { 2 } + 0 \beta _ { 2 } = 0 , } \\
{ \beta _ { 1 } \alpha _ { 3 } + 0 \beta _ { 3 } = 0 , }
\end{array} \quad \text { isto é } \quad \left\{\begin{array}{l}
\beta_{1} \alpha_{2}=0 \\
\beta_{1} \alpha_{3}=0
\end{array}\right.\right.
$$

Portanto, $\alpha_{1}=\alpha_{2}=\alpha_{3}=0$, que faz $\Delta=0$ (contradição). Tudo que foi feito acima para $u v$ pode ser feito, de modo análogo, para $v u, u w, v w, w v$ e assim concluímos que

$$
u v, v u, u w, w u, v w, w v \notin I_{K^{*}}^{3}(G) .
$$

Juntando estes resultados com as congruências (4.5), podemos escrever que

$$
\begin{gathered}
\text { (I) } \quad u v \equiv v u\left(\bmod I_{K^{*}}^{3}(G)\right) \quad \text { e } u v, v u \notin I_{K^{-}}^{3}(G), \\
\text { (II) } \quad u w \equiv v u\left(\bmod I_{K^{-}}^{3}(G)\right) \text { e } u w, w u \notin I_{K^{\prime}}^{3}(G), \\
\text { (III) } \quad v w \equiv v v\left(\bmod I_{K^{-}}^{3}(G)\right) \text { e } v w, w v \notin I_{K^{-}}^{3}(G),
\end{gathered}
$$

onde claramente (I), (II) e (III) verificam as hipóteses da propriedade (ii) da Proposição 2.3.1 das bases multiplicativas filtradas que implica nas igualdades $u v=v u, u w=w u$ e $v w=w v$, isto é, $u, v, w$ comutam entre si, fazendo a álgebra $I_{K}(G)=K[u, v, w]$ comutativa (contradição).

Concluímos pelo acima provado que a álgebra $I G G$ não admite uma base multiplicativa filtrada. 
Como aplicação direta do teorema acima. segue que os seguintes $p$-grupos $G$ com três geradores não admitem uma base multiplicativa filtrada na sua álgebra de grupo $K G$.

(A) 0 2-grupo de ordem 32 dado na apresentação

$$
G_{1}=\left\langle a, b, c, a^{8}=b^{2}=c^{2}=1, a b=b a, a c=c a, b c=c a^{4} b\right\rangle,
$$

onde $(b, c)=a^{4}$.

(B) O p-grupo de ordem $p^{4}$ ( $p$ primo ímpar) definido por

$$
G_{2}=\left\langle a, b, c \mid a^{p^{2}}=b^{p}=c^{p}=1,(b, c)=a^{p},(a, b)=(a, c)=1\right\rangle,
$$

onde $(b, c)=a^{p}$ com $p \geq 3$.

(C) Os p-grupos finitos com subgrupo cíclico de índice $p^{2}$, abaixo indicados, dados na referência [13], calculados por Y. Ninomiya (1994).

(i) O $p$-grupo de ordem $p^{m}$, com $m \geq 4$ e expoente $p^{m-2}$

$$
G_{3}=\left\langle a, b, c \mid a^{p^{m-2}}=1, b^{p}=1, c^{p}=1, a b=b a, c a=a c, c b c^{-1}=a^{p^{m-3}} b\right\rangle,
$$

onde $(c, b)=p^{m-3}$ com $p^{m-3} \geq 3$.

(ii) O 2-grupo de ordem $2^{m}$ e expoente $2^{m-2}, m>4$, onde $(c, b)=a^{2^{m-3}}$.

$$
G_{4}=\left\langle a, b, c \mid a^{2^{m-2}}=1, b^{2}=1, c^{2}=1, a b=b a, a c=c a, c^{-1} b c=a^{2^{m-3}} b\right\rangle
$$

(D) Consideramos agora a classe de todos os grupos finitos $G$ tais que $\frac{G}{Z(G)} \approx C_{p} \times C_{p}$, onde $p$ é um número primo, $Z(G)$ é o centro de $G$ e $C_{p}$ é um grupo cíclico de ordem p. Estes grupos foram estudados por G. Leal e C. Polcino Milies em [15], onde foi provado que $\frac{G}{Z(G)} \approx C_{p} \times C_{p}$ se, e somente se $G=D \times A$, onde $A$ é m grupo 
abeliano e $D$ é um p-grupo indecomponível tal que $D=\langle x, y, Z(D)\rangle \operatorname{com} x y \neq y x$, $x, y \in G$ e $Z(D)$ tem posto menor ou igual a 3. Estudamos aqui o caso onde $G$ é indecomponível, isto é, quando $G=D$ e $A=\{1\}$.

Como estes grupos são da forma $D=\langle x, y, Z(D)\rangle$ e estamos interessados em grupos com três geradores, vamos considerar unicamente o caso em que posto $(Z(D))=1$. O caso $p=2$ está descrito em [10] e existem duas famílias nessas condições:

$$
\begin{aligned}
& \left.D_{1}=\left\langle x, y, t_{1}\right| x^{2}=y^{2}=t_{1}^{2^{m_{1}}}=1,(x, y)=t_{1}^{2^{m_{1}-1}}, t_{1} \text { central }\right\rangle, \\
& \left.D_{2}=\left\langle x, y, t_{1}\right| x^{2}=y^{2}=t_{1}^{2^{m_{1}}}=1,(x, y)=t_{1}^{2^{m_{1}-1}}, t_{1} \text { central }\right\rangle,
\end{aligned}
$$

onde $2^{m_{1}-1} \geq 3$.

O caso $p>2$ foi estudado por C. Polcino Milies e E. Jespers em [11]. Também existem duas famílias:

$$
\begin{aligned}
& \left.D_{3}=\langle x, y, t| x^{p}=y^{p}=t^{p^{n}}=1,[x, y]=t^{p^{n-1}}, t \text { central }\right\rangle, \\
& \left.D_{4}=\langle x, y, t| x^{p}=t, y^{p}=t^{p}=1,[x, y]=t^{p^{n-1}}, t \text { central }\right\rangle,
\end{aligned}
$$

onde $p^{n-1} \geq 3$.

Observação: No caso onde $p=2$ e $m_{1}=1$, temos que $G$ na apresentação $D_{1}$ só pode ser o 2-grupo diedral e na apresentação $D_{2}$ é quatérnio com oito elementos e neste caso as álgebras de grupo modulares coincidentemente possuem bases multiplicativas filtradas para todo corpo $K$ de característica 2 no caso diedral e no caso quatérnio apenas para os corpos $K$ de característica 2 que possuem uma raiz cúbica primitiva da unidade, como provamos no Teorema Principal. 


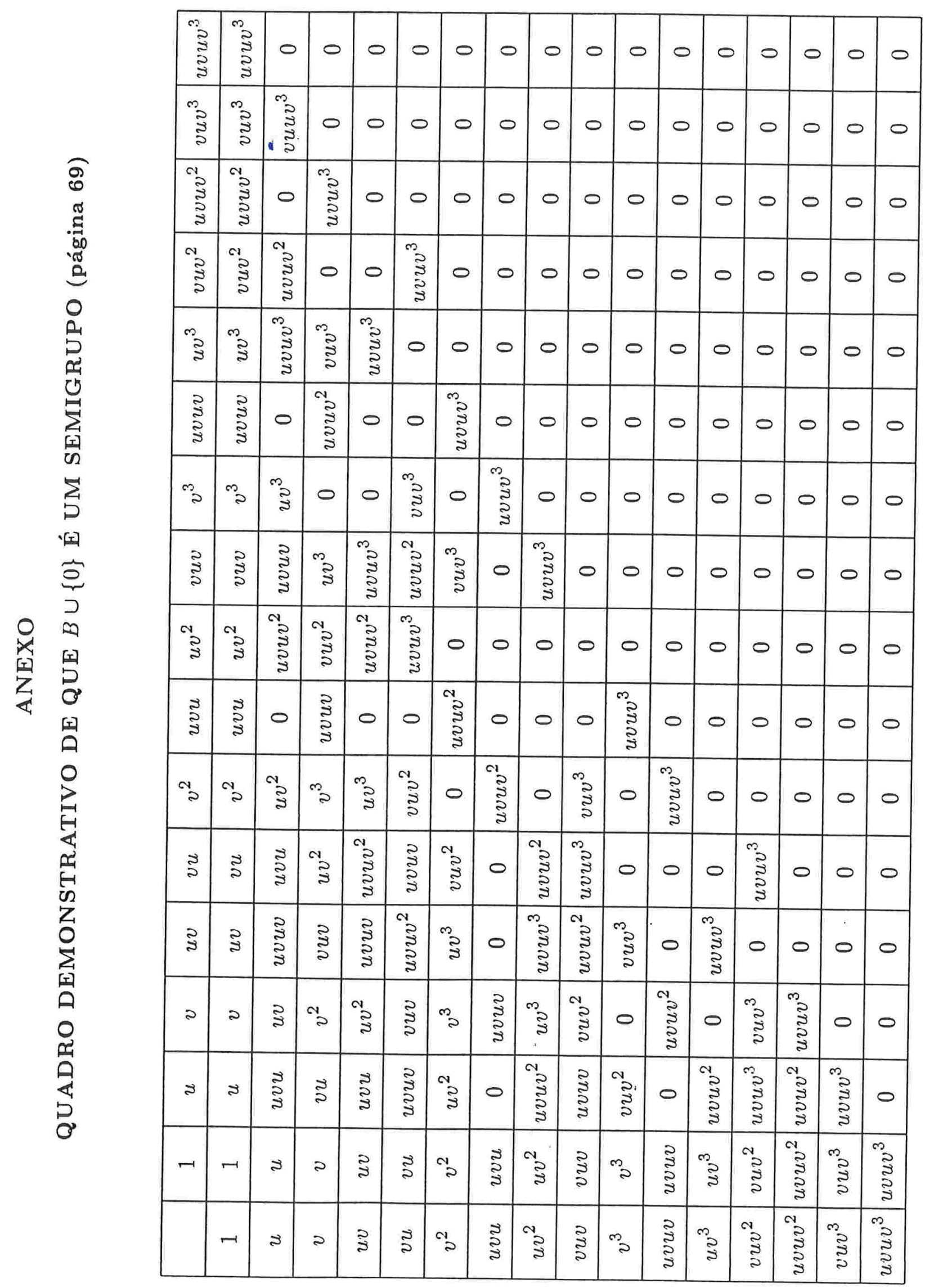




\section{Referências}

[1] Bautista, R., Gabriel, P., Roiter, A., and Salmeron, L. Representation of finite algebras and multiplicative bases. Invent.-Math. 81(2) (1985), 217-285.

[2] Carns, G.L., Chao, C.Y. On the radical of the group algebra of a p-group over a modular field. Proc. Amer. Math. Soc. 33(2) (1972), 323-328.

[3] Coleman, D.B. Finite groups with isomorphic group algebras. Trans. Amer. Soc., 105 (1962), 1-8.

[4] Felzenswalb, B. Álgebras de Dimensão Finita. 12oㅡolóquio Bras. de Matemática (1979).

[5] Gow, R., Huppert, B., Knörr, R., Manz, O., Willens, W. Representation theory in arbitrary characteristic. Casa Editrice Dott. A Milani, Trento (1993).

[6] Hall Jr., M. Teoria de los grupos. Editorial F. Trillas, S.A. (1969), México.

[7] Herstein, I. Non Commutative Rings. John Wiley \& Sons, NY (1968).

[8] Huppert, B. Endliche Gruppen I. Springer-Verlag, Berlin-Heidelberg-New York, 1967. 
[9] Jennings, S.A. The structure of the group ring of a p-group over a modular field. Trans. Amer. Math. Soc., 50 (1941), 175-185.

[10] Jespers, E.; Leal, G.; Polcino Milies, C. Classifying indecomposable R.A. loops. J. of Algebra, 176, 569-584 (1995).

[11] Jespers, E.; Polcino Milies, C. Units of group rings. Journal of Pure and Applied Algebra, 107, 233-251 (1996).

[12] Karpislovsky, G. The Jacobson radical of groups algebras. North-Holland Math. Studies 135. (Notas de Matemática 115.)

[13] Koshitani, S. Group rings of metacyclic p-groups. Proc. Japan Acad., 52, 169-172 (1975).

- [14] Kupisch, H. Symmetrische Algebren mit endlich vielen unzerlegbaren Darstellungen, I. J. Reine Angew. Math. 219 (1965), 1-25.

[15] Leal, G.; Polcino Milies, C. Isomorphic group (and loop) algebras. J. of Algebra, 155 (1993), 195-210.

[16] Lendrock, P, Michler, G.O. Block structure of the smallest Janko group. Math. Ann., 232(3), (1978), 205-238.

[17] Ninomiya, Y. Finite p-groups with cyclic subgroups of index $p^{2}$. Math. J. Okayama Univ., 36 (1994), 1-21.

[18] Paris, L. Some examples of group algebras without filtered multiplicative basis. L'Enseignement Math. 33 (1987), 307-314.

[19] Suzuki, M. Group Theory II. Springer-Verlag, New York, Berlin-Heidelberg, Tokyo, 1986. 